Modeling, Identification and Control, Vol. 35, No. 3, 2014, pp. 169-190, ISSN 1890-1328

\title{
Recent Advances in Static Output-Feedback Controller Design with Applications to Vibration Control of Large Structures
}

\author{
F. Palacios-Quiñonero ${ }^{1}$ J. Rubió-Massegú ${ }^{1}$ J.M. Rossell ${ }^{1}$ H.R. Karimi ${ }^{2}$ \\ ${ }^{1}$ CoDAlab. Department of Applied Mathematics III, Universitat Politècnica de Catalunya (UPC) \\ Av. Bases de Manresa 61-73, 08242-Manresa, Barcelona, Spain \\ E-mail: francisco.palacios@upc.edu, josep.rubio@upc.edu, josep.maria.rossell@upc.edu \\ ${ }^{2}$ Department of Engineering, Faculty of Engineering and Science \\ University of Agder (UiA), N-4898 Grimstad, Norway \\ E-mail: hamid.r.karimi@uia.no
}

\begin{abstract}
In this paper, we present a novel two-step strategy for static output-feedback controller design. In the first step, an optimal state-feedback controller is obtained by means of a linear matrix inequality (LMI) formulation. In the second step, a transformation of the LMI variables is used to derive a suitable LMI formulation for the static output-feedback controller. This design strategy can be applied to a wide range of practical problems, including vibration control of large structures, control of offshore wind turbines, control of automotive suspensions, vehicle driving assistance and disturbance rejection. Moreover, it allows designing decentralized and semi-decentralized static output-feedback controllers by setting a suitable zerononzero structure on the LMI variables. To illustrate the application of the proposed methodology, two centralized static velocity-feedback $H_{\infty}$ controllers and two fully decentralized static velocity-feedback $H_{\infty}$ controllers are designed for the seismic protection of a five-story building.
\end{abstract}

Keywords: Static Output-feedback; Decentralized Control; Structural Vibration Control

\section{Introduction}

Nowadays, a wide variety of sophisticated statefeedback controller designs can be formulated as linear matrix inequality (LMI) optimization problems [Boyd et al. (1994); Huang and Mao (2009); Amato et al. (2010); Oishi and Fujioka (2010); Wang et al. (2010); Dhawan and Kar (2011); Du et al. (2011); Liu et al. (2011); Chen and Wang (2012)], which can be efficiently solved using standard computational tools as those provided by the MATLAB Robust Control Toolbox [Balas et al. (2011)]. Moreover, LMI-based control design strategies allow including complex information constraints by setting a suitable zero-nonzero struc- ture on the LMI optimization variables [Wang et al. (2009)]. This feature can be particularly relevant in control problems with a large number of sensors and actuation devices. In this case, decentralized and semidecentralized strategies help to improve the performance and robustness of the control system by reducing the amount of information exchange and the computational burden in real-time operation [Šiljak (1991); Lunze (1992); Chen and Stanković (2005); Rossell et al. (2010); Zečević and Šiljak (2010); Palacios-Quiñonero et al. (2010, 2011, 2012a); Karimi et al. (2013)].

It must be highlighted, however, that having access to the full state information is a quite uncommon situation in practice and, usually, the information avail- 
able for feedback purposes consists of a reduced set of observed output variables that can be expressed as linear combinations of the states. In this context, static output-feedback controllers constitute a very interesting option [Syrmos et al. (1997); Crusius and Trofino (1999); Prempain and Postlethwaite (2001); Zečević and Siljak (2004); Bara and Boutayeb (2005); Feng et al. (2011)]. In its simplest formulation, a static output-feedback controller can be written in the following form:

$$
u(t)=K y(t),
$$

where $K$ is a constant matrix that allows obtaining the vector of control actions $u(t)$ from the vector of observed outputs $y(t)$ by means of a simple matrix product. The practical advantages provided by this kind of controllers, however, are balanced by the challenging theoretical and computational problems associated with static output-feedback controller design [Moerder and Calise (1985); Cao et al. (1998); Toscano (2006); Gadewadikar et al. (2007); Feng et al. (2011)].

Recently, a two-step computational strategy for static output-feedback controller design was presented in Palacios-Quiñonero et al. (2014b). In the first step, an optimal state-feedback controller is computed by solving an LMI optimization problem $\mathcal{P}_{s}$. Next, the static output-feedback controller is obtained by solving a second LMI optimization problem $\mathcal{P}_{o}$, which is derived from $\mathcal{P}_{s}$ by means of a suitable transformation of the LMI variables [Rubió-Massegú et al. (2013b)]. This approach is conceptually simple and computationally effective, it allows taking advantage of the wide literature on LMI-based state-feedback controller design strategies and, moreover, makes it possible to synthesize static output-feedback controllers with information constraints by setting a proper zero-nonzero structure on the variables of $\mathcal{P}_{o}$.

The definition of the variables transformation used to derive the output-feedback LMI optimization problem $\mathcal{P}_{o}$ involves a constant matrix $L$ that plays an important role in the applicability and effectiveness of the computational strategy. Preliminary works with the simplified choice $L=0$ have produced interesting results in the fields of vibration control of large structures [Rubió-Massegú et al. (2012); Palacios-Quiñonero et al. (2012b,c, 2013, 2014e)], control of offshore wind turbines [Bakka and Karimi (2013); Bakka et al. (2014)], control of active vehicle suspensions [Rubió-Massegú et al. (2013a); Palacios-Quiñonero et al. (2014a)], vehicle driving assistance [Oufroukh and Mammar (2014)] and disturbance rejection [Ballesteros et al. (2013)]. More advanced choices of the $L$ matrix, motivated by the theoretical results in Palacios-Quiñonero et al. (2014b), have been recently applied to the fields of vibration control of large structures [Palacios-Quiñonero et al. (2014c)] and control of vehicle suspensions [Rubió-Massegú et al. (2014)] with positive results.

This paper makes a twofold contribution: Firstly, we provide a complete, well structured and practical presentation of the main elements involved in the two-step design strategy for output-feedback controller design. Secondly, we introduce an advanced $L$ matrix choice that allows computing fully decentralized velocity-feedback $H_{\infty}$ controllers for vibration control of large structures. To enhance the practical aspects, the theoretical exposition is complemented with the design of four static velocity-feedback $H_{\infty}$ controllers for the seismic protection of a five-story building. These controllers include centralized and fully decentralized designs and illustrate in detail the usage of null and advanced $L$-matrices.

For clarity and simplicity, the discussion has been restricted to the continuous-time $H_{\infty}$ approach. However, it has to be highlighted that the proposed design methodology can also be applied to other control strategies that admit an LMI formulation. For example, discrete-time $H_{\infty}$ applications can be found in Palacios-Quiñonero et al. (2012b, 2014a); Ballesteros et al. (2013), and continuous-time energy-to-peak applications have been presented in Palacios-Quiñonero et al. (2014c,e)

The paper is organized as follows: Sect. 2 provides a detailed description of the state-feedback LMI optimization problem $\mathcal{P}_{s}$ and the derivation of the outputfeedback LMI optimization problem $\mathcal{P}_{o}$. In Sect.3, the two-step procedure for output-feedback controller design is presented, and some relevant aspects associated with the usage of null and advanced $L$-matrices are discussed. In Sect. 4, a mathematical model of a five-story building subject to seismic disturbances and equipped with a full set of interstory actuation devices is introduced. For the seismic protection of this building, three different controllers are designed in Sect. 5: an optimal state-feedback $H_{\infty}$ controller and two centralized velocity-feedback $H_{\infty}$ controllers, which are computed using a null $L$-matrix and the advanced $L$ matrix choice proposed in Palacios-Quiñonero et al. (2014b). In Sect.6, information constraints are discussed and two fully decentralized velocity-feedback $H_{\infty}$ controllers are designed, using a null $L$-matrix and a new $L$-matrix choice specially devised for the $H_{\infty}$ approach. To demonstrate the effectiveness of the proposed static velocity-feedback controllers, numerical simulations of the five-story building vibrational response are conducted in Sect. 7 , using the full scale North-South El Centro 1940 and North-South Kobe 1995 seismic records as ground acceleration inputs. Finally, in Sect. 8, some conclusions and future research lines are briefly presented. 


\section{Theoretical background}

In this section, we introduce some fundamental concepts, results and notations that will be used throughout the paper. First, the main elements of statefeedback $H_{\infty}$ controller design are summarized. Next, the general results presented in Rubió-Massegú et al. (2013b) are applied to the particular case of static output-feedback $H_{\infty}$ controller design.

\subsection{State-feedback $H_{\infty}$ controller}

Let us consider the system

$$
\mathcal{S}:\left\{\begin{aligned}
\dot{x}(t) & =A x(t)+B_{u} u(t)+B_{w} w(t), \\
z(t) & =C_{z} x(t)+D_{z} u(t)
\end{aligned}\right.
$$

where $x(t) \in \mathbb{R}^{n}$ is the state, $u(t) \in \mathbb{R}^{m}$ is the control input, $w(t) \in \mathbb{R}^{r}$ is the disturbance input, $z(t) \in \mathbb{R}^{d}$ is the controlled output and $A, B_{u}, B_{w}, C_{z}$ and $D_{z}$ are constant matrices with appropriate dimensions. For a given static state-feedback controller

$$
u(t)=G x(t),
$$

with state gain matrix $G \in \mathbb{R}^{m \times n}$, we obtain the following closed-loop system:

$$
\mathcal{S}_{\mathrm{cl}}:\left\{\begin{array}{l}
\dot{x}(t)=A_{G} x(t)+B_{w} w(t) \\
z_{G}(t)=C_{G} x(t)
\end{array}\right.
$$

where the matrices $A_{G}$ and $C_{G}$ have the form

$$
A_{G}=A+B_{u} G, \quad C_{G}=C_{z}+D_{z} G
$$

The $H_{\infty}$-norm of the state-feedback controller defined by the control gain matrix $G$ is the largest energy gain from the disturbance input to the controlled output

$$
\gamma_{G}=\sup _{\|w\|_{2} \neq 0} \frac{\left\|z_{G}\right\|_{2}}{\|w\|_{2}}
$$

where $\|\cdot\|_{2}$ is the usual continuous 2-norm

$$
\|f\|_{2}=\left[\int_{0}^{\infty}\{f(t)\}^{T} f(t) d t\right]^{1 / 2} .
$$

The value $\gamma_{G}$ can be computed using the closed-loop transfer function from the disturbance input $w(t)$ to the controlled output $z_{G}(t)$

$$
T_{G}(s)=C_{G}\left(s I-A_{G}\right)^{-1} B_{w} .
$$

More precisely, the value $\gamma_{G}$ can be expressed as the $H_{\infty}$-norm of $T_{G}$

$$
\gamma_{G}=\left\|T_{G}\right\|_{\infty}=\sup _{f} \sigma_{\max }\left[T_{G}(2 \pi f j)\right],
$$

where $j=\sqrt{-1}, f$ is the frequency in $\mathrm{Hz}$ and $\sigma_{\max }[\cdot]$ denotes the maximum singular value.

In the $H_{\infty}$ approach, the objective consists of obtaining a control gain matrix $\widetilde{G}$ that produces an asymptotically stable closed-loop matrix $A_{\widetilde{G}}$ and, simultaneously, attains an optimally small $H_{\infty}$-norm $\gamma_{\widetilde{G}}$. An effective method to compute the optimal gain matrix $\widetilde{G}$ is based on the Bounded Real Lemma [Boyd et al. (1994)] which, for a prescribed $\gamma>0$, states the equivalence of the following conditions:

1. $\left\|T_{G}\right\|_{\infty}<\gamma$, and $A_{G}$ is asymptotically stable.

2. There exists a symmetric positive-definite matrix $X \in \mathbb{R}^{n \times n}$ such that the matrix inequality

$$
\left[\begin{array}{cr}
A_{G} X+X A_{G}^{T}+\gamma^{-2} B_{w} B_{w}^{T} & * \\
C_{G} X & -I
\end{array}\right]<0
$$

holds, where * denotes the transpose of the element in the symmetric position and the matrix inequality indicates that the corresponding matrix is negative definite.

From eqs. (5) and (10), we obtain the nonlinear matrix inequality

$$
\left[\begin{array}{cr}
A X+X A^{T}+B_{u} G X+X G^{T} B_{u}^{T}+\gamma^{-2} B_{w} B_{w}^{T} & * \\
C_{z} X+D_{z} G X & -I
\end{array}\right]<0
$$

which, by introducing the new variables

$$
Y=G X, \quad \eta=\gamma^{-2}
$$

can be converted into the following LMI:

$$
\left[\begin{array}{cr}
A X+X A^{T}+B_{u} Y+Y^{T} B_{u}^{T}+\eta B_{w} B_{w}^{T} & * \\
C_{z} X+D_{z} Y & -I
\end{array}\right]<0 .
$$

The optimal state-feedback $H_{\infty}$ controller can now be computed by solving the following LMI optimization problem:

$$
\mathcal{P}_{s}: \begin{cases}\text { maximize } & \eta \\ \text { subject to } & X>0, \eta>0 \\ & \text { and the LMI in eq. (13) }\end{cases}
$$

where the matrices $X$ and $Y$ are the optimization variables. If this optimization problem attains an optimal value $\widetilde{\eta}_{s}$ for the pair of matrices $\left(\widetilde{X}_{s}, \widetilde{Y}_{s}\right)$, then the gain matrix

$$
\widetilde{G}_{s}=\widetilde{Y}_{s} \widetilde{X}_{s}^{-1}
$$

defines a state-feedback controller

$$
u(t)=\widetilde{G}_{s} x(t)
$$




$$
\left[\begin{array}{cr}
A Q X_{Q} Q^{T}+Q X_{Q} Q^{T} A^{T}+A R X_{R} R^{T}+R X_{R} R^{T} A^{T}+B_{u} Y_{R} R^{T}+R Y_{R}^{T} B_{u}^{T}+\eta B_{w} B_{w}^{T} & * \\
C_{z} Q X_{Q} Q^{T}+C_{z} R X_{R} R^{T}+D_{z} Y_{R} R^{T} & -I
\end{array}\right]<0
$$

Figure 1: Linear matrix inequality for the static output-feedback $H_{\infty}$ controller design

with an asymptotically stable closed-loop matrix $A_{\widetilde{G}_{s}}$, and an optimal $H_{\infty}$-norm

$$
\gamma_{\widetilde{G}_{s}}=\left(\widetilde{\eta}_{s}\right)^{-1 / 2}
$$

Remark 1 For a given state gain matrix $\widehat{G}$, the $\gamma$ value corresponding to the state-feedback controller

$$
u(t)=\widehat{G} x(t)
$$

can be computed by considering the closed-loop matrices

$$
A_{\widehat{G}}=A+B_{u} \widehat{G}, \quad C_{\widehat{G}}=C_{z}+D_{z} \widehat{G},
$$

and the LMI

$$
\left[\begin{array}{cr}
A_{\widehat{G}} X+X\left(A_{\widehat{G}}\right)^{T}+\eta B_{w} B_{w}^{T} & * \\
C_{\widehat{G}} X & -I
\end{array}\right]<0 .
$$

If the auxiliary optimization problem

$$
\mathcal{P}_{a}: \begin{cases}\text { maximize } & \eta \\ \text { subject to } & X>0, \eta>0 \\ & \text { and the LMI in eq. (20) }\end{cases}
$$

attains an optimal value $\widetilde{\eta}_{a}$, then $A_{\widehat{G}}$ is asymptotically stable and the $H_{\infty}$-norm of the controller in eq. (18) has the value

$$
\gamma_{\widehat{G}}=\left(\widetilde{\eta}_{a}\right)^{-1 / 2}
$$

Note that the matrices $A_{\widehat{G}}$ and $C_{\widehat{G}}$ have fixed values in the problem $\mathcal{P}_{a}$ and, consequently, the matrix inequality in eq. (20) is linear. Moreover, if the LMI optimization problem $\mathcal{P}_{s}$ admits an optimal solution $\widetilde{G}_{s}$, then we have the inequality

$$
\gamma_{\widetilde{G}_{s}} \leq \gamma_{\widehat{G}}
$$

\subsection{Static output-feedback $H_{\infty}$ controllers}

Let us consider the vector of observed outputs $y(t) \in$ $\mathbb{R}^{p}$ that can be written in the form

$$
y(t)=C_{y} x(t)
$$

where $C_{y} \in \mathbb{R}^{p \times n}$ is a matrix with row-rank $p<n$. In this case, we are interested in obtaining a static output-feedback controller of the form

$$
u(t)=K y(t),
$$

which uses a constant gain matrix $K \in \mathbb{R}^{m \times p}$ to compute the control actions $u(t)$ from the observed-output information $y(t)$. By substituting eq. (24) into eq. (25), we obtain the associated state-feedback controller

$$
u(t)=G_{K} x(t),
$$

with state gain matrix

$$
G_{K}=K C_{y}
$$

The $H_{\infty}$-norm of the static output-feedback controller in eq. (25) can be defined using the associated state gain matrix $G_{K}$ as follows:

$$
\gamma_{K}=\gamma_{G_{K}}=\left\|T_{G_{K}}\right\|_{\infty}
$$

and the problem of designing an optimal static outputfeedback $H_{\infty}$ controller can be seen as a constrained state-feedback control problem, where the state gain matrix $G$ must admit the factorization $G=K C_{y}$. More precisely, a static output-feedback $H_{\infty}$ controller of the form given in eq. (25) can be computed by solving the following optimization problem:

$$
\mathcal{P}_{c}: \begin{cases}\text { maximize } & \eta \\ \text { subject to } & X>0, \eta>0,(X, Y) \in \mathcal{M}, \\ & \text { and the LMI in eq. }(13),\end{cases}
$$

where $\mathcal{M}$ is a set that contains all the pairs of matrices $(X, Y)$ for which there exists a matrix $K \in \mathbb{R}^{m \times p}$ such that

$$
Y X^{-1}=K C_{y}
$$

An effective computational strategy to deal with the non-convex optimization problem $\mathcal{P}_{c}$ has been formulated in Rubió-Massegú et al. (2013b). This strategy introduces the following transformations of the LMI variables:

$$
X=Q X_{Q} Q^{T}+R X_{R} R^{T}, \quad Y=Y_{R} R^{T},
$$

where $Q \in \mathbb{R}^{n \times(n-p)}$ is a matrix whose columns are a basis of $\operatorname{Ker}\left(C_{y}\right)$, and $R \in \mathbb{R}^{n \times p}$ has the following form:

$$
R=C_{y}^{\dagger}+Q L
$$

where

$$
C_{y}^{\dagger}=C_{y}^{T}\left(C_{y} C_{y}^{T}\right)^{-1}
$$


is the Moore-Penrose pseudoinverse of $C_{y}$, and $L \in$ $\mathbb{R}^{(n-p) \times p}$ is a constant matrix. The transformations in eq. (31) introduce, as new LMI variables, two symmetric matrices $X_{Q} \in \mathbb{R}^{(n-p) \times(n-p)}$ and $X_{R} \in \mathbb{R}^{p \times p}$, and a general matrix $Y_{R} \in \mathbb{R}^{m \times p}$.

By substituting the variable transformations given in eq. (31) into the LMI in eq. (13), we obtain the LMI presented in Fig. 1 and, according to the results obtained in Rubió-Massegú et al. (2013b), a static output-feedback controller can be computed by solving the following LMI optimization problem:

$$
\mathcal{P}_{o}: \begin{cases}\text { maximize } & \eta \\ \text { subject to } & X_{Q}>0, X_{R}>0, \eta>0 \\ & \text { and the LMI in Fig. } 1 .\end{cases}
$$

If the LMI optimization problem $\mathcal{P}_{o}$ can be properly solved, producing an optimal value $\widetilde{\eta}_{o}$ for the triplet $\left(\widetilde{X}_{Q}, \widetilde{X}_{R}, \widetilde{Y}_{R}\right)$, then the output gain matrix

$$
\widetilde{K}=\widetilde{Y}_{R}\left(\widetilde{X}_{R}\right)^{-1}
$$

defines a static output-feedback controller

$$
u(t)=\widetilde{K} y(t)
$$

with asymptotically stable closed-loop matrix

$$
A_{\widetilde{K}}=A+B_{u} \widetilde{K} C_{y}
$$

and an associated $H_{\infty}$-norm $\gamma_{\widetilde{K}}$ that satisfies

$$
\gamma_{\widetilde{K}} \leq \widetilde{\gamma}_{o}=\left(\widetilde{\eta}_{o}\right)^{-1 / 2}
$$

Remark 2 As indicated in eq. (38), the LMI optimization problem $\mathcal{P}_{o}$ only provides an upper bound of the $\gamma$ value corresponding to the static output-feedback controller given in eq. (36). According to the discussion in Remark 1 , the actual value of $\gamma_{\widetilde{K}}$ can be computed by considering the associated state-feedback gain matrix

$$
G_{\widetilde{K}}=\widetilde{K} C_{y}
$$

and solving the LMI optimization problem $\mathcal{P}_{a}$ in eq. (21) with $\widehat{G}=G_{\widetilde{K}}$. If this auxiliary LMI optimization problem attains the optimal value $\widetilde{\eta}_{a}$, then the $H_{\infty}$-norm of the output-feedback controller in eq. (36) satisfies

$$
\gamma_{\widetilde{K}}=\left(\widetilde{\eta}_{a}\right)^{-1 / 2}
$$

Moreover, looking at eqs. (23) and (38), we also have the inequality

$$
\gamma_{\widetilde{G}_{s}} \leq \gamma_{\widetilde{K}} \leq \widetilde{\gamma}_{o}
$$

\section{Design strategies}

The LMI variable transformations defined in eqs. (31) (33) contain a constant matrix $L$, which can provide an important design flexibility and, at the same time, can exert a critical influence in the applicability of the design strategy. In this section, we take advantage of this property to define a versatile and effective twostep design procedure for static output-feedback $H_{\infty}$ controllers. First, the two-step design procedure is presented. Next, some feasibility issues associated with the case $L=0$ are discussed and a numerical line of solution is proposed. Finally, some recent theoretical results obtained in Palacios-Quiñonero et al. (2014b) are applied to introduce a more advanced choice of the $L$-matrix.

\subsection{General two-step design procedure}

Considering the discussion presented in Sect. 2, the output-feedback $H_{\infty}$ controller design procedure can be summarized as follows:

\section{Step 1. State-feedback $H_{\infty}$ controller design}

(S1.1) Solve the optimization problem $\mathcal{P}_{s}$ in eq. (14) to compute the optimal state gain matrix $\widetilde{G}_{s}$ and the optimal cost $\gamma_{\widetilde{G}_{s}}$.

(S1.2) Check that the design requirements are satisfied by the state-feedback controller $u(t)=$ $\widetilde{G}_{s} x(t)$.

\section{Step 2. Output-feedback $H_{\infty}$ controller design}

(S2.1) Choose a suitable $L$-matrix to define the $R$ matrix in eq. (32) and formulate the LMI optimization problem $\mathcal{P}_{o}$ in eq. (34).

(S2.2) Solve the optimization problem $\mathcal{P}_{o}$ to compute the output gain matrix $\widetilde{K}$ and the upper bound $\widetilde{\gamma}_{o}$.

(S2.3) Compare the optimal cost $\gamma_{\widetilde{G}_{s}}$ and the suboptimal output-feedback cost $\gamma_{\widetilde{K}}$.

(S2.4) Assess the performance of the output-feedback controller $u(t)=\widetilde{K} y(t)$, taking as a reference the optimal state-feedback controller obtained in Step 1.

Remark 3 In Step 1, we assume that a satisfactory state-feedback controller can be designed for the problem under consideration. We also assume that this state-feedback controller can be computed by solving an LMI optimization problem. These two conditions are positively satisfied in a large number of practical 


$$
\left[\begin{array}{cr}
\widehat{A} Q X_{Q} Q^{T}+Q X_{Q} Q^{T} \widehat{A}^{T}+\widehat{A} R X_{R} R^{T}+R X_{R} R^{T} \widehat{A}^{T}+B_{u} Y_{R} R^{T}+R Y_{R}^{T} B_{u}^{T}+\eta B_{w} B_{w}^{T} & * \\
C_{z} Q X_{Q} Q^{T}+C_{z} R X_{R} R^{T}+D_{z} Y_{R} R^{T} & -I
\end{array}\right]<0
$$

Figure 2: Perturbed linear matrix inequality for static output-feedback $H_{\infty}$ controller design

control problems, for which effective LMI-based statefeedback controller design strategies can be found in the literature.

Remark 4 Step 1 can be considered as an exploratory step. The state-feedback controller has a complete access to the state information. If no suitable controller can be found under full-information conditions, then the possibility of obtaining a satisfactory static outputfeedback controller should be seriously reconsidered.

Remark 5 The underlying idea in the proposed design procedure consists of obtaining a static outputfeedback controller that satisfies $\gamma_{\widetilde{K}} \approx \gamma_{\widetilde{G}_{\odot}}$. It has been observed in practice that, frequently, the performance of these almost optimal output-feedback controllers is very similar to the performance of the optimal state-feedback controller. Accordingly, the optimal cost $\gamma_{\widetilde{G}_{s}}$ and the output-feedback cost $\gamma_{\widetilde{K}}$ are compared in step (S2.3) to assess the degree of optimality attained by the output-feedback controller. To this end, an approximate comparison can be made using the upper bound $\widetilde{\gamma}_{o}$ in eq. (38) and the inequality in eq. (41). When a more precise comparison is necessary, the actual value of $\gamma_{\widetilde{K}}$ can be computed by solving the auxiliary LMI optimization problem $\mathcal{P}_{a}$ discussed in Remark 2. It should be highlighted that the optimal state-feedback controller and the static outputfeedback controller are both designed using the same controlled output $z(t)=C_{z} x(t)+D_{z} u(t)$ and, consequently, the $\gamma$-value comparison is meaningful.

\subsection{Design strategy with $L=0$}

From an algebraic point of view, the choice $L=0$ is a natural option that produces the simplified $R$-matrix

$$
R=C_{y}^{\dagger}
$$

in the design step (S2.1). In this case, no information from the optimal state-feedback controller is used to compute the static output-feedback controller. This approach has been applied in Rubió-Massegú et al. (2013a) to design an output-feedback $H_{\infty}$ controller for vehicle suspensions with positive results. The choice $L=0$ has also been successfully used to compute static velocity-feedback controllers for seismic protection of large structures in Rubió-Massegú et al. (2012);
Palacios-Quiñonero et al. (2012b,c, 2014e). In these applications to structural vibration control, however, the output-feedback LMI optimization problem $\mathcal{P}_{o}$ is initially reported to be unfeasible by the MATLAB LMI solver and the design step (S2.2) cannot be completed.

To overcome the encountered feasibility issues, we introduce a slightly perturbed state matrix

$$
\widehat{A}=A-\epsilon I_{n},
$$

where $\epsilon$ is a small positive number, and consider the following LMI optimization problem:

$$
\widehat{\mathcal{P}}_{o}: \begin{cases}\text { maximize } & \eta \\ \text { subject to } X_{Q}>0, X_{R}>0, \eta>0, & \text { and the LMI in Fig. } 2 .\end{cases}
$$

In all the aforementioned applications to structural vibration control, the MATLAB LMI solver fails to solve the corresponding LMI optimization problem $\mathcal{P}_{o}$ but, quite surprisingly, it has no difficulties to deal with the perturbed LMI problem $\widehat{\mathcal{P}}_{o}$. Taking advantage of this computational trick, we can solve the LMI problem $\widehat{\mathcal{P}}_{o}$ to obtain an optimal triplet $\left(\widehat{X}_{Q}, \widehat{X}_{R}, \widehat{Y}_{R}\right)$ with an associated cost $\widehat{\eta}_{o}$. We can also obtain the output gain matrix

$$
\widehat{K}=\widehat{Y}_{R}\left(\widehat{X}_{R}\right)^{-1}
$$

and define the static output-feedback controller

$$
u(t)=\widehat{K} y(t)
$$

with associated state-feedback matrix

$$
G_{\widehat{K}}=\widehat{K} C_{y} .
$$

Remark 6 It should be noted that the value

$$
\widehat{\gamma}_{o}=\left(\widehat{\eta}_{o}\right)^{-1 / 2}
$$

is not an upper bound of $\gamma_{\widehat{K}}$ and can only be considered as an estimate. Additionally, the feasibility of the perturbed LMI problem $\widehat{\mathcal{P}}_{o}$ neither implies the asymptotic stability of the closed-loop state matrix

$$
A_{\widehat{K}}=A+B_{u} \widehat{K} C_{y} .
$$

To give a proper response to these issues, we can follow the ideas presented in Remark 1 and consider the LMI 
optimization problem $\mathcal{P}_{a}$ in eq. (21) with $\widehat{G}=G_{\widehat{K}}$. If this auxiliary LMI problem can be solved and attains the optimal value $\widehat{\eta}_{a}$, then the closed-loop matrix $A_{\widehat{K}}$ is asymptotically stable and the actual $H_{\infty}$-norm of the static output-feedback controller defined by the output gain matrix $\widehat{K}$ can be computed as

$$
\gamma_{\widehat{K}}=\left(\widehat{\eta}_{a}\right)^{-1 / 2}
$$

Remark 7 The feasibility issues mentioned in this section constitute a strange and poorly understood phenomenon. From a practical perspective, extensive numerical simulations show that using a perturbed state matrix of the form given in eq. (43) is an effective strategy to overcome the problem. This line of solution with $\epsilon=0.01$ is used in Sect. 5.2 and Sect. 6.1 with positive results.

\subsection{Advanced choice of the $L$-matrix}

The choice $L=0$ has the obvious advantage of simplicity. However, this option also has the important drawback of ignoring the particular characteristics of the problem under consideration. In order to obtain more advantageous choices of the matrix $L$, the properties of the LMI variable transformations in eqs. (31)-(33) have been investigated in detail. Next, we summarize some results presented in Palacios-Quiñonero et al. (2014b), which lead to a more advanced choice of the matrix $L$.

For a given matrix $L \in \mathbb{R}^{(n-p) \times p}$, let us consider the variety of matrices

$$
\mathcal{V}_{L}=\left\{X=Q X_{Q} Q^{T}+R X_{R} R^{T} \mid X_{Q} \in \mathbb{P}_{n-p}, X_{R} \in \mathbb{P}_{p}\right\},
$$

where $\mathbb{P}_{k}=\left\{X \in \mathbb{S}^{k \times k} \mid X>0\right\}$ denotes the set of all $k \times k$ symmetric positive-definite matrices. The following assertions hold (for details, see Theorem 1 and Theorem 2 in Palacios-Quiñonero et al. (2014b)):

1. The family $\mathcal{V}=\left\{\mathcal{V}_{L} \mid L \in \mathbb{R}^{(n-p) \times p}\right\}$ defines a partition of $\mathbb{P}_{n}$.

2. For a given $X \in \mathbb{P}_{n}$, there exists a unique $L_{X} \in$ $\mathbb{R}^{(n-p) \times p}$ such that $X \in \mathcal{V}_{L_{X}}$. This unique $L$ matrix can be explicitly written in the following form:

$$
L_{X}=Q^{\dagger} X C_{y}^{T}\left(C_{y} X C_{y}^{T}\right)^{-1}
$$

where $Q^{\dagger}=\left(Q^{T} Q\right)^{-1} Q^{T}$ denotes the MoorePenrose pseudoinverse of $Q$.

After selecting a particular matrix $L$, the LMI optimization problem $\mathcal{P}_{o}$ in eq. (34) can be seen as a constrained version of the LMI optimization problem $\mathcal{P}_{c}$ in eq. (29) with the additional condition $X \in \mathcal{V}_{L}$. The expression in eq. (51) suggests a natural choice of the matrix $L$, which consists of selecting the $L$-matrix corresponding to the optimal $X$-matrix obtained in the state-feedback problem $\mathcal{P}_{s}$. More precisely, if an optimal solution to the problem $\mathcal{P}_{s}$ in the design step (S1.1) has been attained with the optimal LMI matrices $\left(\widetilde{X}_{s}, \widetilde{Y}_{s}\right)$, then we choose the following $L$-matrix in step $(\mathrm{S} 2.1)$ :

$$
\widetilde{L}=Q^{\dagger} \widetilde{X}_{s} C_{y}^{T}\left(C_{y} \widetilde{X}_{s} C_{y}^{T}\right)^{-1}
$$

and solve the output-feedback LMI optimization problem $\mathcal{P}_{o}$ in step (S2.2) using the $R$-matrix

$$
\widetilde{R}=C_{y}^{\dagger}+Q \widetilde{L}
$$

This advanced choice of the $L$-matrix has been used in Palacios-Quiñonero et al. (2014b) and PalaciosQuiñonero et al. (2014d) to design static velocityfeedback $H_{\infty}$ controllers for seismic protection of large structures. According to the results reported in these works, the new approach has a double advantage: (i) it can help to avoid the feasibility issues associated with the choice $L=0$ and, simultaneously, (ii) it can also help to reduce the value $\gamma_{\widetilde{K}}$ producing, in some cases, static output-feedback controllers that are practically optimal.

\section{Five-story building mathematical model}

In this section, we provide a state-space model for the lateral motion of a five-story building, which will be used in the controller designs presented in Sect. 5 and Sect. 6 and the numerical simulations conducted in Sect.7. The building motion can be described by the second-order differential equation

$$
M \ddot{q}(t)+C_{d} \dot{q}(t)+K_{s} q(t)=T_{u} u(t)+T_{w} w(t),
$$

where

$$
q(t)=\left[q_{1}(t), q_{2}(t), q_{3}(t), q_{4}(t), q_{5}(t)\right]^{T}
$$

is the vector of displacements relative to the ground and $q_{i}(t)$, for $i=1 \ldots 5$, represents the lateral displacement of the $i$ th story $s_{i}$ with respect to the ground level $s_{0}$. The vector of control actions is

$$
u(t)=\left[u_{1}(t), u_{2}(t), u_{3}(t), u_{4}(t), u_{5}(t)\right]^{T} .
$$

The control action $u_{i}(t)$ is applied by the actuation device $a_{i}$, which produces a pair of opposite structural forces as indicated in Fig. 3. The seismic ground acceleration is denoted by $w(t) . M, C_{d}$, and $K_{s}$ are the mass, damping and stiffness matrices, respectively. $T_{u}$ 


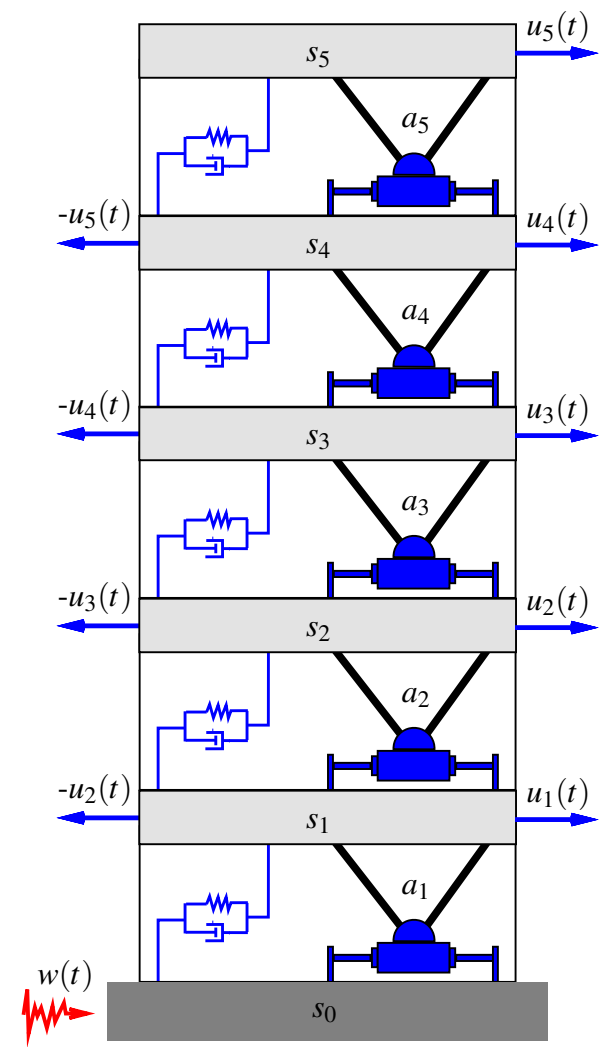

Figure 3: Five-story building mechanical model: actuation scheme and external disturbance

is the control location matrix and $T_{w}$ is the excitation location matrix. In the different controllers designs and numerical simulations conducted in this paper, the following particular values of the mass, damping and stiffness matrices have been used:

$$
\begin{gathered}
M=10^{3} \times\left[\begin{array}{ccccc}
215.2 & 0 & 0 & 0 & 0 \\
0 & 209.2 & 0 & 0 & 0 \\
0 & 0 & 207.0 & 0 & 0 \\
0 & 0 & 0 & 204.8 & 0 \\
0 & 0 & 0 & 0 & 266.1
\end{array}\right], \quad(57) \\
C_{d}=10^{3} \times\left[\begin{array}{cccccc}
260.2 & -92.4 & 0 & 0 & 0 \\
-92.4 & 219.6 & -81.0 & 0 & 0 \\
0 & -81.0 & 199.5 & -72.8 & 0 \\
0 & 0 & -72.8 & 186.7 & -68.7 \\
0 & 0 & 0 & -68.7 & 127.4
\end{array}\right], \\
K_{s}=10^{6} \times\left[\begin{array}{rrrrr}
260 & -113 & 0 & 0 & 0 \\
-113 & 212 & -99 & 0 & 0 \\
0 & -99 & 188 & -89 & 0 \\
0 & 0 & -89 & 173 & -84 \\
0 & 0 & 0 & -84 & 84
\end{array}\right], \quad(59)
\end{gathered}
$$

where masses are in $\mathrm{kg}$, damping coefficients in $\mathrm{Ns} / \mathrm{m}$, and stiffness coefficients in $\mathrm{N} / \mathrm{m}$. The mass and stiffness values are similar to those presented in Kurata et al. (1999), and $C_{d}$ is a Rayleigh damping matrix with a $2 \%$ damping ratio on the first and fifth modes
[Chopra (2007)]. The control location matrix, corresponding to the actuation scheme depicted in Fig. 3, and the excitation location matrix have the following form:

$$
T_{u}=\left[\begin{array}{rrrrr}
1 & -1 & 0 & 0 & 0 \\
0 & 1 & -1 & 0 & 0 \\
0 & 0 & 1 & -1 & 0 \\
0 & 0 & 0 & 1 & -1 \\
0 & 0 & 0 & 0 & 1
\end{array}\right], T_{w}=-M\left[\begin{array}{l}
1 \\
1 \\
1 \\
1 \\
1
\end{array}\right] .
$$

By considering the vector of interstory drifts

$$
r(t)=\left[q_{1}, q_{2}-q_{1}, q_{3}-q_{2}, q_{4}-q_{3}, q_{5}-q_{4}\right]^{T},
$$

and the state vector

$$
x(t)=\left[\begin{array}{c}
r(t) \\
\dot{r}(t)
\end{array}\right],
$$

we can derive a first-order state-space model

$$
\dot{x}(t)=A x(t)+B_{u} u(t)+B_{w} w(t)
$$

with

$$
\begin{gathered}
A=P A_{I} P^{-1}, B_{u}=P\left(B_{u}\right)_{I}, B_{w}=P\left(B_{w}\right)_{I}, \\
A_{I}=\left[\begin{array}{cc}
{[0]_{5 \times 5}} & I_{5} \\
-M^{-1} K_{s} & -M^{-1} C_{d}
\end{array}\right], \\
\left(B_{u}\right)_{I}=\left[\begin{array}{c}
{[0]_{5 \times 5}} \\
M^{-1} T_{u}
\end{array}\right], \quad\left(B_{w}\right)_{I}=\left[\begin{array}{r}
{[0]_{5 \times 1}} \\
-[1]_{5 \times 1}
\end{array}\right],
\end{gathered}
$$

where $[0]_{n \times m}$ represents a zero-matrix of the indicated dimensions, $I_{n}$ is the identity matrix of order $n,[1]_{n \times 1}$ denotes a vector of dimension $n$ with all its entries equal to 1 , and $P$ is the change of basis matrix

$$
P=\left[\begin{array}{rrrrrrrrrr}
1 & 0 & 0 & 0 & 0 & 0 & 0 & 0 & 0 & 0 \\
-1 & 1 & 0 & 0 & 0 & 0 & 0 & 0 & 0 & 0 \\
0 & -1 & 1 & 0 & 0 & 0 & 0 & 0 & 0 & 0 \\
0 & 0 & -1 & 1 & 0 & 0 & 0 & 0 & 0 & 0 \\
0 & 0 & 0 & -1 & 1 & 0 & 0 & 0 & 0 & 0 \\
0 & 0 & 0 & 0 & 0 & 1 & 0 & 0 & 0 & 0 \\
0 & 0 & 0 & 0 & 0 & -1 & 1 & 0 & 0 & 0 \\
0 & 0 & 0 & 0 & 0 & 0 & -1 & 1 & 0 & 0 \\
0 & 0 & 0 & 0 & 0 & 0 & 0 & -1 & 1 & 0 \\
0 & 0 & 0 & 0 & 0 & 0 & 0 & 0 & -1 & 1
\end{array}\right]
$$

corresponding to the state transformation

$$
\left[\begin{array}{c}
r(t) \\
\dot{r}(t)
\end{array}\right]=P\left[\begin{array}{c}
q(t) \\
\dot{q}(t)
\end{array}\right] .
$$

For the particular building matrices $M, C_{d}, K_{s}, T_{u}$ and $T_{w}$ given in eqs. (57)-(60), the values of the corresponding system matrices $A, B_{u}$ and $B_{w}$ are displayed in Fig. 4. 


$$
\begin{aligned}
& A=10^{3} \times\left[\begin{array}{ccccccccccc}
0 & 0 & 0 & 0 & 0 & 0.0010 & 0 & 0 & 0 & 0 \\
0 & 0 & 0 & 0 & 0 & 0 & 0.0010 & 0 & 0 & 0 \\
0 & 0 & 0 & 0 & 0 & 0 & 0 & 0.0010 & 0 & 0 \\
0 & 0 & 0 & 0 & 0 & 0 & 0 & 0 & 0.0010 & 0 \\
0 & 0 & 0 & 0 & 0 & 0 & 0 & 0 & 0 & 0.0010 \\
-0.6831 & 0.5251 & 0 & 0 & 0 & -0.0008 & 0.0004 & 0 & 0 & 0 \\
0.6831 & -1.0652 & 0.4732 & 0 & 0 & 0.0006 & -0.0011 & 0.0004 & 0 & 0 \\
0 & 0.5402 & -0.9515 & 0.4300 & 0 & 0 & 0.0004 & -0.0010 & 0.0004 & 0 & 0 \\
0 & 0 & 0.4783 & -0.8645 & 0.4102 & 0 & 0 & 0.0004 & -0.0009 & 0.0003 \\
0 & 0 & 0 & 0.4346 & -0.7258 & 0 & 0 & 0 & 0.0004 & -0.0008
\end{array}\right], \\
& B_{u}=10^{-5} \times\left[\begin{array}{ccccc}
0 & 0 & 0 & 0 & 0 \\
0 & 0 & 0 & 0 & 0 \\
0 & 0 & 0 & 0 & 0 \\
0 & 0 & 0 & 0 & 0 \\
0 & 0 & 0 & 0 & 0 \\
0.4647 & -0.4647 & 0 & 0 & 0 \\
-0.4647 & 0.9427 & -0.4780 & 0 & 0 \\
0 & -0.4780 & 0.9611 & -0.4831 & 0 \\
0 & 0 & -0.4831 & 0.9714 & -0.4883 \\
0 & 0 & 0 & -0.4883 & 0.8641
\end{array}\right], \quad B_{w}=\left[\begin{array}{c}
0 \\
0 \\
0 \\
0 \\
0 \\
-1 \\
0 \\
0 \\
0 \\
0
\end{array}\right] .
\end{aligned}
$$

Figure 4: System matrices of the first-order model with interstory drifts and interstory velocities as state variables

\section{Centralized controllers}

In this section, the design strategies discussed in Sect. 3 are applied to compute centralized velocity-feedback $H_{\infty}$ controllers for the seismic protection of the fivestory building presented in Sect. 4. The controller designs and numerical simulations are conducted with the system

$$
\mathcal{S}:\left\{\begin{array}{l}
\dot{x}(t)=A x(t)+B_{u} u(t)+B_{w} w(t), \\
z(t)=C_{z} x(t)+D_{z} u(t)
\end{array}\right.
$$

defined by the system matrices $A, B_{u}$ and $B_{w}$ given in Fig. 4, and the controlled output matrices

$$
C_{z}=\left[\begin{array}{c}
I_{10} \\
{[0]_{5 \times 10}}
\end{array}\right], \quad D_{z}=10^{-6.3}\left[\begin{array}{c}
{[0]_{10 \times 5}} \\
I_{5}
\end{array}\right] .
$$

All the computations have been carried out with MAT$\mathrm{LAB}$ and a relative error of $10^{-6}$ has been set in the function mincx () to solve the different LMI optimization problems.

\subsection{Centralized state-feedback controller}

According to the general design procedure presented in Sect 3.1, we begin by obtaining a suitable statefeedback $H_{\infty}$ controller, which uses the full state information to compute the control actions and plays a twofold role in the design procedure: (i) serving as a natural reference in the performance assessment of the output-velocity controllers and (ii) providing an appropriate $X$-matrix for the advanced choices of the $L$ matrix. As indicated in step (S1.1), we solve the LMI optimization problem $\mathcal{P}_{s}$ in eq. (14) with the matrices $A, B_{u}, B_{w}, C_{z}, D_{z}$ given in Fig. 4 and eq. (70), obtaining an optimal state-feedback $H_{\infty}$ controller

$$
u(t)=\widetilde{G}_{s} x(t)
$$

with an associated $\gamma$-value

$$
\gamma_{\widetilde{G}_{s}}=0.7466
$$

The state gain matrix $\widetilde{G}_{s}$ and the corresponding optimal matrix $\widetilde{X}_{s}$ are displayed in Fig. 5 .

To demonstrate the good behavior of the statefeedback controller defined by the gain matrix $\widetilde{G}_{s}$, the maximum singular values of the closed-loop pulse transfer function

$$
T_{\widetilde{G}_{s}}(2 \pi f j)=C_{\widetilde{G}_{s}}\left(2 \pi f j I-A_{\widetilde{G}_{s}}\right)^{-1} B_{w}
$$

and the open-loop pulse transfer function

$$
T(2 \pi f j)=C_{z}(2 \pi f j I-A)^{-1} B_{w}
$$

are presented in Fig. 6. The graphic of the open-loop transfer function (dash-dotted black line) shows the frequency response characteristics of the uncontrolled building. In particular, it can be clearly appreciated the building resonant frequencies, which are located at $1.0082,2.8246,4.4929,5.7974$, and $6.7735 \mathrm{~Hz}$. The graphic of the closed-loop transfer function $T_{\widetilde{G}_{s}}(2 \pi f j)$ (solid blue line) shows the ability of the state-feedback $H_{\infty}$ controller to mitigate the building vibrational response at the resonant frequencies. This graphic has 


$$
\begin{aligned}
& \widetilde{G}_{s}=10^{7} \times\left[\begin{array}{rrrrrrrrrr}
-0.4776 & 0.2153 & -0.0534 & -0.0054 & 0.0166 & -0.3387 & -0.0845 & -0.0678 & -0.0446 & -0.0235 \\
0.3146 & -0.1359 & -0.3397 & 0.1789 & 0.0198 & -0.1190 & -0.3263 & -0.1172 & -0.0576 & -0.0280 \\
0.5122 & 0.0742 & -0.4065 & -0.0928 & 0.1560 & -0.0972 & -0.1233 & -0.3229 & -0.0818 & -0.0239 \\
0.1288 & 0.0336 & 0.8589 & -1.3026 & 0.6093 & -0.0718 & -0.0778 & -0.0670 & -0.3510 & -0.0167 \\
0.0293 & 0.0453 & 0.0710 & 1.0589 & -1.5497 & -0.0468 & -0.0422 & -0.0338 & -0.0110 & -0.3683
\end{array}\right] \\
& \widetilde{X}_{s}=10^{3} \times\left[\begin{array}{rrrrrrrrrr}
0.0070 & -0.0008 & -0.0011 & -0.0010 & -0.0006 & -0.0267 & 0.0320 & 0.0022 & -0.0006 & -0.0003 \\
-0.0008 & 0.0085 & -0.0008 & -0.0012 & -0.0008 & 0.0204 & -0.0552 & 0.0381 & 0.0017 & 0.0010 \\
-0.0011 & -0.0008 & 0.0100 & -0.0009 & -0.0006 & 0.0018 & 0.0225 & -0.0633 & 0.0401 & 0.0038 \\
-0.0010 & -0.0012 & -0.0009 & 0.0126 & -0.0007 & 0.0014 & 0.0011 & 0.0259 & -0.0657 & 0.0350 \\
-0.0006 & -0.0008 & -0.0006 & -0.0007 & 0.0124 & 0.0012 & -0.0005 & -0.0010 & 0.0300 & -0.0593 \\
-0.0267 & 0.0204 & 0.0018 & 0.0014 & 0.0012 & 5.0702 & -4.7629 & -0.5036 & 0.0432 & -0.0552 \\
0.0320 & -0.0552 & 0.0225 & 0.0011 & -0.0005 & -4.7629 & 9.3260 & -4.2863 & -0.4632 & -0.0425 \\
0.0022 & 0.0381 & -0.0633 & 0.0259 & -0.0010 & -0.5036 & -4.2863 & 9.8315 & -5.2443 & 0.0163 \\
-0.0006 & 0.0017 & 0.0401 & -0.0657 & 0.0300 & 0.0432 & -0.4632 & -5.2443 & 11.3930 & -5.9711 \\
-0.0003 & 0.0010 & 0.0038 & 0.0350 & -0.0593 & -0.0552 & -0.0425 & 0.0163 & -5.9711 & 10.5260
\end{array}\right] .
\end{aligned}
$$

Figure 5: State-feedback gain matrix and $X$-matrix corresponding to the optimal state-feedback $H_{\infty}$ controller

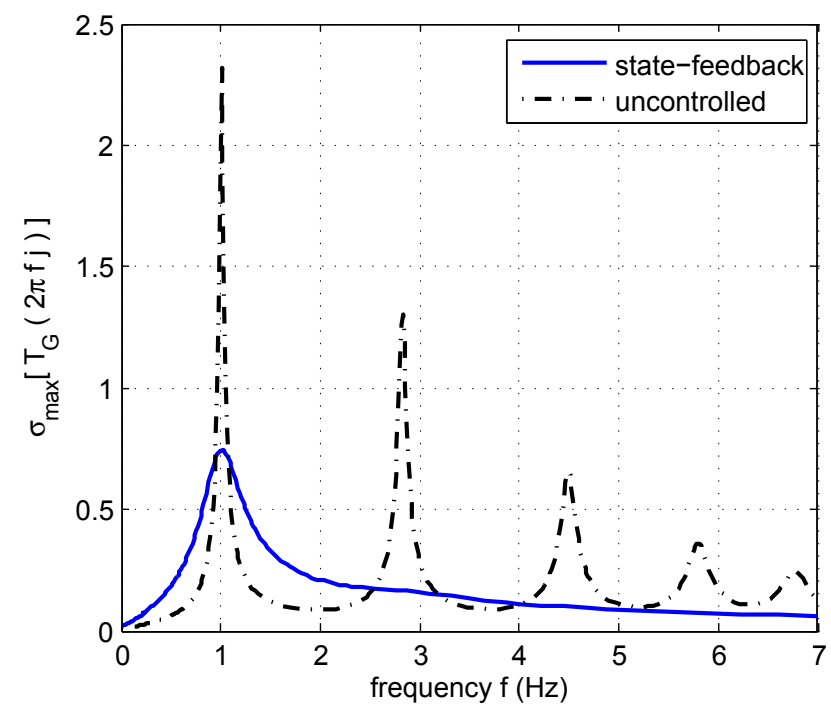

Figure 6: Maximum singular values for the closed-loop pulse transfer function $T_{\widetilde{G}_{s}}(2 \pi f j)$ (solid blue line) and the open-loop transfer function $T(2 \pi f j)$ (dash-dotted black line)

a single peak, whose magnitude corresponds to the $\gamma$ value given in eq. (72).

To provide a more complete picture of the statefeedback controller performance, we have also conducted numerical simulations of the five-story building vibrational response, using the full scale North-South El Centro 1940 seismic record as ground acceleration input (see Fig. 7). The maximum absolute interstory drifts are displayed in Fig. 8(a), where the blue line with circles corresponds to the state-feedback $H_{\infty}$ con-

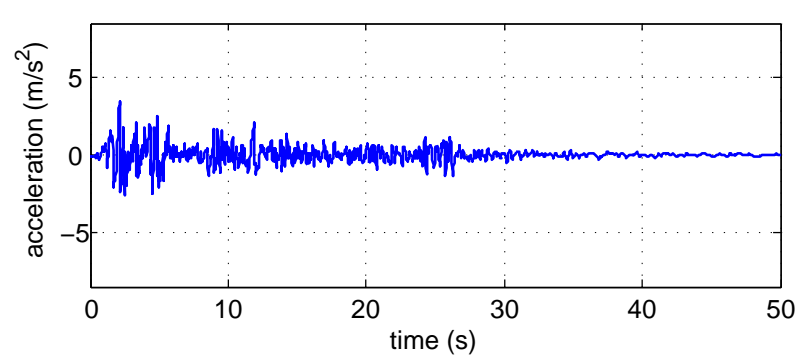

Figure 7: Full scale North-South El Centro 1940 seismic record, with an absolute acceleration peak of $3.42 \mathrm{~m} / \mathrm{s}^{2}$

troller and the black line with rectangles presents the vibrational response of the uncontrolled building. The maximum absolute control efforts corresponding to the state-feedback controller are displayed in Fig. 8(b). A quick look at the graphics clearly shows that the proposed state-feedback $H_{\infty}$ controller attains a good level of reduction in the interstory drift peak-values with moderate levels of control effort. In what follows, we will assume that $\widetilde{G}_{s}$ defines a suitable statefeedback controller for the five-story building introduced in Sect. 4.

Remark 8 It has been observed that solving the LMI optimization problem $\mathcal{P}_{s}$ in eq. (14) using different MATLAB versions can produce small differences in the matrix $\widetilde{X}_{s}$ and substantial differences in some elements of the control gain matrix $\widetilde{G}_{s}$. These differences have also been observed when using the same MATLAB version on different computers. However, in all the cases the same optimal value $\gamma_{\widetilde{G}_{s}}=0.7466$ has been obtained, and the corresponding graphics of singular 

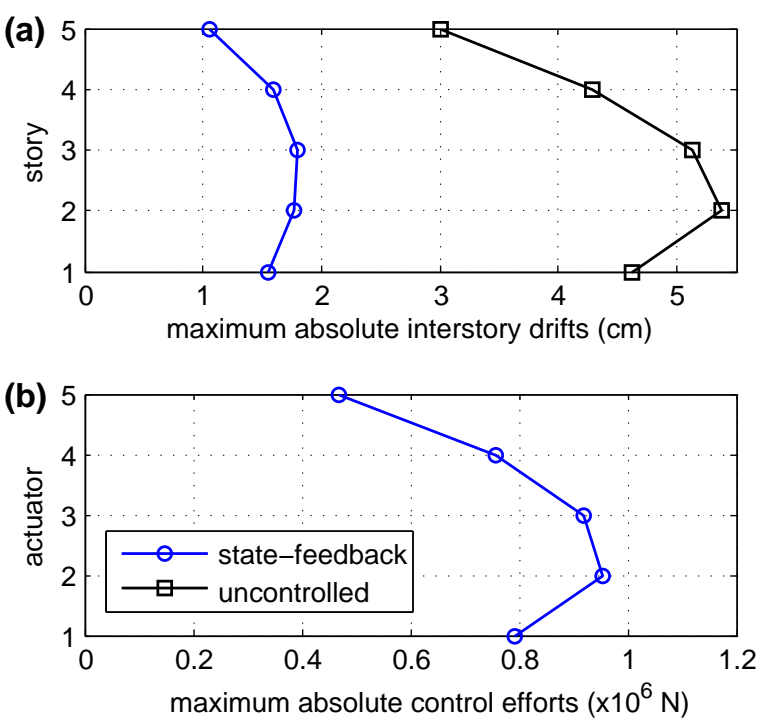

Figure 8: (a) Maximum absolute interstory drifts and (b) maximum absolute control efforts corresponding to the uncontrolled building (black line with rectangles) and the optimal statefeedback $H_{\infty}$ controller (blue line with circles). The full scale North-South El Centro 1940 seismic record has been used as ground acceleration disturbance

values, interstory drift peak-values and control effort peak-values are practically identical to those shown in Figs. 6, 8(a) and 8(b), respectively.

\subsection{Centralized velocity-feedback controller with $L=0$}

Now, we use the design methodology proposed in Sect. 3.2 to obtain a centralized static velocity-feedback $H_{\infty}$ controller. To this end, we consider an outputfeedback controller

$$
u(t)=K y(t),
$$

where the observed-output is the vector of interstory velocities

$$
y(t)=\left[\dot{r}_{1}(t), \dot{r}_{2}(t), \dot{r}_{3}(t), \dot{r}_{4}(t), \dot{r}_{5}(t)\right]^{T},
$$

which can be written in the form

$$
y(t)=C_{y} x(t)
$$

with

$$
C_{y}=\left[[0]_{5 \times 5} \quad I_{5}\right] .
$$

To complete the design step (S2.1), we take a basis of $\operatorname{Ker}\left(C_{y}\right)$ and obtain the matrix

$$
Q=\left[\begin{array}{c}
I_{5} \\
{[0]_{5 \times 5}}
\end{array}\right]
$$

and, by setting $L=0$ in eq. (32), we also obtain the $R$-matrix

$$
R_{0}=\left[\begin{array}{c}
{[0]_{5 \times 5}} \\
I_{5}
\end{array}\right]
$$

As indicated in Sect. 3.2, the LMI optimization problem $\mathcal{P}_{o}$ corresponding to the matrices $Q$ and $R$ given in eqs. (79) and (80) is reported to be infeasible by the MATLAB LMI solver and produces no positive results. To overcome this difficulty, we consider the perturbed state matrix

$$
\widehat{A}=A-0.01 I_{10}
$$

and solve the perturbed LMI optimization problem $\widehat{\mathcal{P}}_{o}$ in eq. (44). In this case, no feasibility issues are encountered and we obtain the velocity gain matrix

$$
\begin{gathered}
\widehat{K}=10^{6} \times \\
{\left[\begin{array}{rrrrr}
-5.5401 & -0.7411 & 0.3965 & 0.2060 & 0.1017 \\
-0.7496 & -5.6394 & 1.0210 & 0.4926 & 0.1729 \\
0.7814 & 0.7110 & -6.8120 & -0.3484 & 0.2644 \\
0.3852 & 0.4091 & -0.1740 & -5.7953 & -0.2354 \\
0.1804 & 0.2339 & 0.3265 & -0.2358 & -6.2418
\end{array}\right],}
\end{gathered}
$$

with an estimated $H_{\infty}$-norm

$$
\widehat{\gamma}_{o}=0.7794 \text {. }
$$

To guarantee the asymptotic stability and compute the actual $H_{\infty}$-norm of the static velocity-feedback controller

$$
u(t)=\widehat{K} y(t)
$$

we solve the auxiliary LMI problem $\mathcal{P}_{a}$ discussed in Remark 6, obtaining the $\gamma$-value

$$
\gamma_{\widehat{K}}=0.7539
$$

Comparing $\gamma_{\widehat{K}}$ with the optimal $\gamma$-value in eq. (72), we can see that the $H_{\infty}$-norm increment produced by the velocity-feedback controller in eq. (84) is inferior to $1 \%$. Moreover, looking at the plots of singular values displayed in Fig. 9, it can also be appreciated that the response similarities between the proposed velocityfeedback controller and the optimal state-feedback controller in eq. (71) are not restricted to the peak value, but rather encompasses the full frequency range.

\subsection{Centralized velocity-feedback controller with advanced $L$-matrix}

Following the ideas presented in Sect. 3.3, we now consider the matrix $C_{y}$ in eq. (78), the matrix $Q$ in eq. (79), the optimal matrix $\widetilde{X}_{s}$ displayed in Fig. 5 and the expression

$$
\widetilde{L}=Q^{\dagger} \widetilde{X}_{s} C_{y}^{T}\left(C_{y} \widetilde{X}_{s} C_{y}^{T}\right)^{-1}
$$




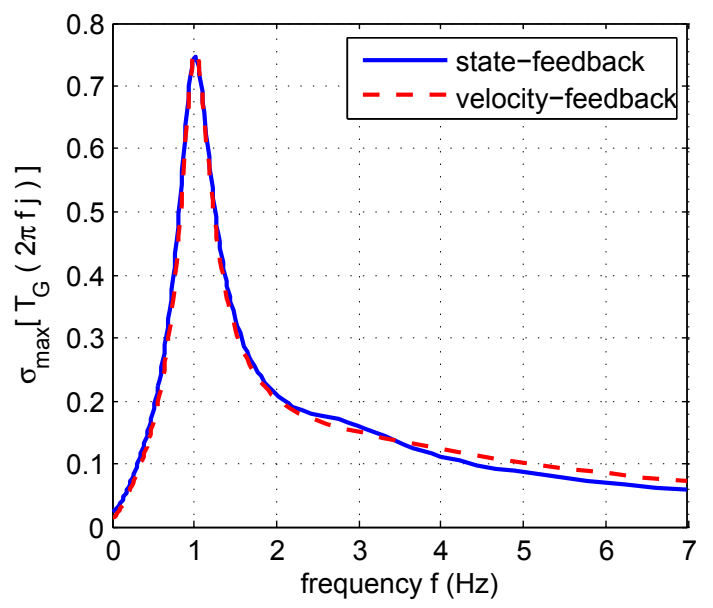

Figure 9: Centralized velocity-feedback controller with $L=0$. Maximum singular values of the closed-loop pulse transfer functions corresponding to the centralized velocity-feedback controller $u(t)=\widehat{K} y(t)$ (red dashed line) and the optimal state-feedback controller $u(t)=$ $\widetilde{G}_{s} x(t)$ (blue solid line)

to compute the $L$-matrix

$\widetilde{L}=\left[\begin{array}{rrrrr}0.0028 & 0.0080 & 0.0062 & 0.0045 & 0.0025 \\ 0.0013 & -0.0034 & 0.0039 & 0.0026 & 0.0016 \\ -0.0023 & -0.0021 & -0.0073 & 0.0004 & 0.0006 \\ -0.0039 & -0.0041 & -0.0039 & -0.0085 & -0.0015 \\ -0.0034 & -0.0035 & -0.0033 & -0.0028 & -0.0073\end{array}\right]$

which produces the $R$-matrix

$$
\widetilde{R}=\left[\begin{array}{c}
\widetilde{L} \\
I_{5}
\end{array}\right] .
$$

Next, we solve the LMI optimization problem $\mathcal{P}_{o}$ corresponding to the matrix $Q$ in eq. (79) and the matrix $R$ given in eq. (88), obtaining the following velocityfeedback gain matrix:

$$
\begin{gathered}
\widetilde{K}=10^{6} \times \\
\left.\qquad \begin{array}{rrrrr}
-2.7372 & -0.9491 & -0.5118 & -0.9908 & -0.7746 \\
-1.0267 & -3.2689 & -1.1102 & -0.6310 & -0.4555 \\
-1.2465 & -0.1394 & -5.3464 & 0.6938 & -0.0092 \\
-1.4048 & -1.7622 & 1.2444 & -3.7717 & -0.2013 \\
-0.9325 & -0.8565 & -0.0779 & -0.0167 & -2.6777
\end{array}\right],
\end{gathered}
$$

with an associated $\gamma$-value that satisfies

$$
\gamma_{\widetilde{K}} \leq 0.7467 .
$$

It is worth highlighting that the advanced choice of the $L$-matrix in eq. (86) allows solving the LMI optimization problem $\mathcal{P}_{o}$ with no feasibility issues. Moreover,

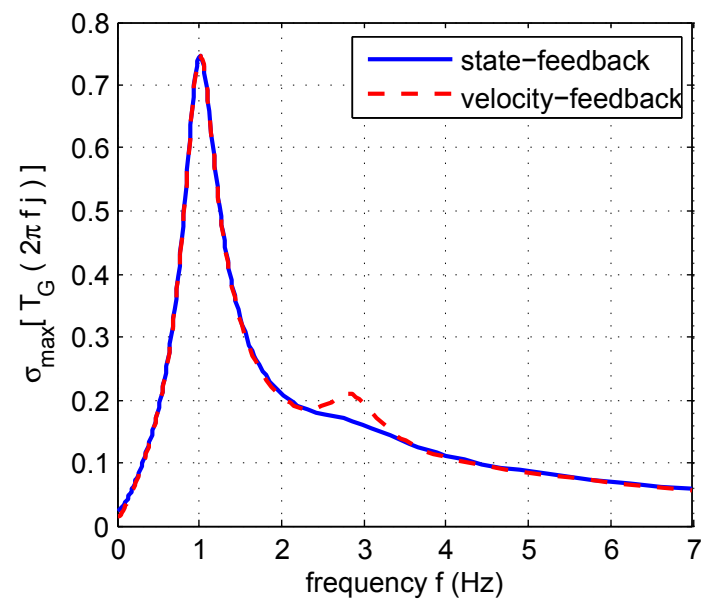

Figure 10: Centralized velocity-feedback controller with $L=\widetilde{L}$. Maximum singular values of the closed-loop pulse transfer functions corresponding to the centralized velocityfeedback controller $u(t)=\widetilde{K} y(t)$ (red dashed line) and the optimal state-feedback controller $u(t)=\widetilde{G}_{s} x(t)$ (blue solid line)

the $\gamma$-value in eq. (72) and the upper bound in eq. (90) indicate that the velocity-feedback controller

$$
u(t)=\widetilde{K} y(t)
$$

is practically optimal. Looking at the plots of singular values displayed in Fig. 10, we can appreciate that the graphic corresponding to the velocity-feedback controller in eq. (91) presents a small peak around the second resonant frequency $(2.8246 \mathrm{~Hz})$. For all the other frequencies, the proposed velocity-feedback controller has virtually the same behavior as the optimal statefeedback controller in eq. (71).

\section{Structured velocity-feedback controllers}

Control gain matrices with a particular zero-nonzero structure can be used to define decentralized or semidecentralized controllers, which can be operated using restricted local feedback information. For vibration control of large structures, fully decentralized static velocity-feedback controllers constitute a case of singular interest. This kind of controllers can be defined by a diagonal output gain matrix and can have the outstanding property of admitting a passive implementation [Palacios-Quiñonero et al. (2012c)]. The main objective of this section is to obtain fully decentralized velocity-feedback controllers of the following form:

$$
u(t)=K^{(d)} y(t)
$$




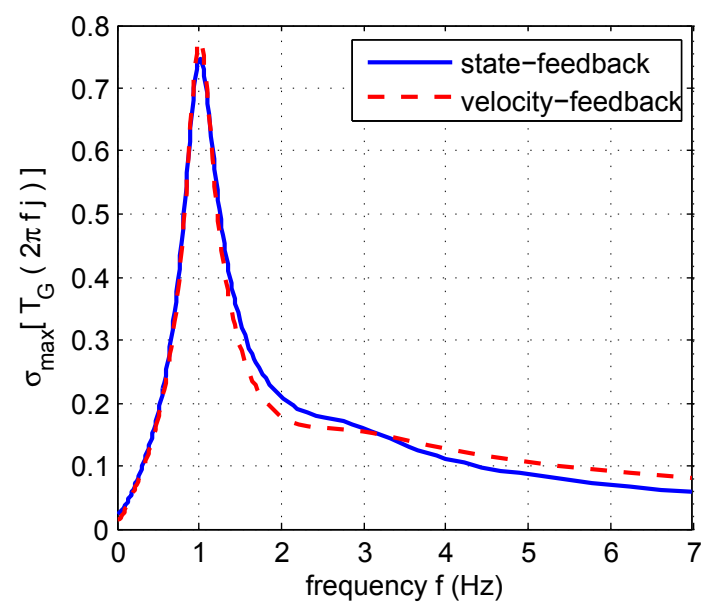

Figure 11: Decentralized velocity-feedback controller with $L=0$. Maximum singular values of the closed-loop pulse transfer functions corresponding to the decentralized velocityfeedback controller $u(t)=\widehat{K}^{(d)} y(t)$ (red dashed line) and the optimal state-feedback controller $u(t)=\widetilde{G}_{s} x(t)$ (blue solid line)

where $y(t)$ is the vector of interstory velocities and $K^{(d)}$ is a diagonal matrix. Considering the explicit expression of the output-feedback gain matrix in eq. (35), this goal can be achieved by solving the LMI optimization problem $\mathcal{P}_{o}$ in eq. (34) with the additional zero-nonzero structure constraints

$$
\begin{gathered}
X_{R}=\left[\begin{array}{lllll}
\mathbf{a} & 0 & 0 & 0 & 0 \\
0 & \mathbf{\square} & 0 & 0 & 0 \\
0 & 0 & \mathbf{\square} & 0 & 0 \\
0 & 0 & 0 & \mathbf{\square} & 0 \\
0 & 0 & 0 & 0 & \mathbf{\square}
\end{array}\right], \\
Y_{R}=\left[\begin{array}{lllll}
\mathbf{\square} & 0 & 0 & 0 & 0 \\
0 & \mathbf{\square} & 0 & 0 & 0 \\
0 & 0 & \mathbf{\square} & 0 & 0 \\
0 & 0 & 0 & \mathbf{\square} & 0 \\
0 & 0 & 0 & 0 & \mathbf{\square}
\end{array}\right],
\end{gathered}
$$

where the black squares represent the allowed positions for nonzero elements.

\subsection{Decentralized velocity-feedback controller with $L=0$}

In the decentralized design, the choice $L=0$ leads us to the same initial feasibility issues discussed in Sect. 5.2. However, as it happened in the centralized case, these difficulties can be overpassed by considering the perturbed state matrix $\widehat{A}$ in eq. (81) and by solving the perturbed LMI optimization problem $\widehat{\mathcal{P}}_{o}$ in eq. (44) with the additional zero-nonzero structure constraints

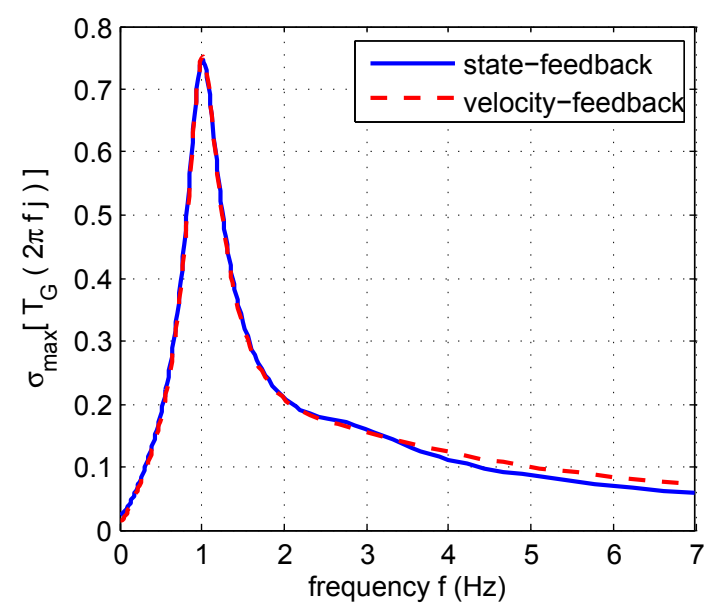

Figure 12: Decentralized velocity-feedback controller with $L=L_{\delta^{*}, \mu^{*}}^{(d)}$. Maximum singular values of the closed-loop pulse transfer functions corresponding to the decentralized velocityfeedback controller $u(t)=\widetilde{K}^{(d)} y(t)$ (red dashed line) and the optimal state-feedback controller $u(t)=\widetilde{G}_{s} x(t)$ (blue solid line)

given in eqs. (93) and (94). As a result, we obtain the diagonal velocity gain matrix

$$
\begin{gathered}
\widehat{K}^{(d)}=10^{6} \times \\
{\left[\begin{array}{ccccc}
-8.0247 & 0 & 0 & 0 & 0 \\
0 & -5.4060 & 0 & 0 & 0 \\
0 & 0 & -4.3416 & 0 & 0 \\
0 & 0 & 0 & -3.6926 & 0 \\
0 & 0 & 0 & 0 & -3.3789
\end{array}\right],}
\end{gathered}
$$

with an estimated $H_{\infty}$-norm

$$
\widehat{\gamma}_{o}^{(d)}=0.7816 \text {. }
$$

Moreover, the resolution of the auxiliary LMI problem $\mathcal{P}_{a}$ in eq. (21) with

$$
\widehat{G}=\widehat{K}^{(d)} C_{y}
$$

guarantees the asymptotic stability of the decentralized static velocity-feedback controller

$$
u(t)=\widehat{K}^{(d)} y(t)
$$

and produces the $\gamma$-value

$$
\gamma_{\widehat{K}^{(d)}}=0.7768 .
$$

In this case, we obtain a $\gamma$-value increment of $4.05 \%$ with respect to the optimal value $\gamma_{\widetilde{G}_{s}}=0.7466$ attained by the state-feedback controller in eq. (71). Despite this $\gamma$-value increment, the plots of singular values in Fig. 11 show that the overall behavior of the decentralized velocity-feedback controller in eq. (98) is still 
quite similar to the behavior exhibited by the optimal state-feedback controller, especially if we consider the magnitude of the uncontrolled frequency response displayed by the dash-dotted black line in Fig. 6 .

\subsection{Decentralized velocity-feedback $H_{\infty}$ controller with advanced $L$-matrix}

In contrast to what happens in the $L=0$ case, the $L$-matrix choice used in Sect. 5.3 to obtain centralized static velocity-feedback controllers produces no positive results in decentralized designs. However, we have observed that satisfactory decentralized controllers can be designed by considering slightly variations of the matrix $\widetilde{L}$ in eq. (52). In particular, a fully decentralized static velocity-feedback energy-to-peak controller with excellent properties has been computed in PalaciosQuiñonero et al. (2014c) using the $L$-matrix

$$
\widetilde{L}_{e t p}^{(d)}=Q^{\dagger} \widetilde{X}_{s} C_{y}^{T}\left(C_{y} \widetilde{X}_{s}^{(d)} C_{y}^{T}\right)^{-1}
$$

where $\widetilde{X}_{s}$ is the optimal $X$-matrix corresponding to the optimal state-feedback controller obtained in the design step $(\mathrm{S} 1.1)$ and $\widetilde{X}_{s}^{(d)}$ is a diagonal matrix that contains the diagonal elements of $\widetilde{X}_{s}$.

In this section, we present a new $L$-matrix choice suitable for decentralized velocity-feedback controllers under the $H_{\infty}$ approach. To this end, we consider the following family of tridiagonal matrices:

$$
\widetilde{X}_{\delta, \mu}^{(t d)}=\delta\left\{\widetilde{X}_{s}^{(d)}+\mu\left[\widetilde{X}_{s}^{(d 1)}+\left(\widetilde{X}_{s}^{(d 1)}\right)^{T}\right]\right\}
$$

where $\delta$ and $\mu$ are real numbers and $\widetilde{X}_{s}^{(d 1)}$ is a matrix that contains the above 1-diagonal of $\widetilde{X}_{s}$. More precisely, denoting by $\widetilde{x}_{i, j}$ and $\widetilde{x}_{i, j}^{(d 1)}$ the elements of $\widetilde{X}_{s}$ and $\widetilde{X}_{s}^{(d 1)}$, respectively, we have:

$$
\begin{cases}\widetilde{x}_{i, i+1}^{(d 1)}=\widetilde{x}_{i, i+1} & \text { for } i=1, \ldots, n-1, \\ \widetilde{x}_{i, j}^{(d 1)}=0 & \text { otherwise. }\end{cases}
$$

From the matrix $\widetilde{X}_{\delta, \mu}^{(t d)}$, we define the $L$-matrix

$$
L_{\delta, \mu}^{(d)}=Q^{\dagger} \widetilde{X}_{s} C_{y}^{T}\left(C_{y} \widetilde{X}_{\delta, \mu}^{(t d)} C_{y}^{T}\right)^{-1}
$$

which allows completing the design steps (S2.1) and (S2.2) with the zero-nonzero structure constraints given in eqs. (93) and (94) for different values of $\delta$ and $\mu$. In our five-story building control problem, we select the particular values

$$
\delta^{*}=0.14, \quad \mu^{*}=0.85
$$

which produce the $L$-matrix

$$
\begin{aligned}
& L_{\delta, \mu^{*}}^{(d)}= \\
& \quad\left[\begin{array}{rrrrr}
-0.0224 & 0.0191 & 0.0109 & 0.0049 & 0.0021 \\
0.0025 & -0.0329 & 0.0210 & 0.0123 & 0.0066 \\
0.0072 & 0.0059 & -0.0370 & 0.0150 & 0.0098 \\
0.0059 & 0.0049 & 0.0038 & -0.0371 & 0.0059 \\
0.0020 & 0.0005 & -0.0001 & 0.0010 & -0.0397
\end{array}\right] .
\end{aligned}
$$

By completing the design steps (S2.1) and (S2.2) with the matrix $L_{\delta^{*} \mu^{*}}^{(d)}$ and the structure constraints in eqs. (93) and (94), we obtain the control gain matrix

$$
\begin{aligned}
\tilde{K}^{(d)} & =10^{6} \times \\
& {\left[\begin{array}{ccccc}
-6.3640 & 0 & 0 & 0 & 0 \\
0 & -5.7753 & 0 & 0 & 0 \\
0 & 0 & -5.3282 & 0 & 0 \\
0 & 0 & 0 & -5.2014 & 0 \\
0 & 0 & 0 & 0 & -6.1716
\end{array}\right] }
\end{aligned}
$$

and the upper bound

$$
\gamma_{\widetilde{K}^{(d)}} \leq 0.7560
$$

which indicates that the decentralized velocityfeedback controller

$$
u(t)=\widetilde{K}^{(d)} y(t)
$$

produces a $\gamma$-value increment inferior to $1.26 \%$ with respect to the optimal value $\gamma_{\widetilde{G}_{s}}=0.7466$. In fact, by solving the LMI optimization problem $\mathcal{P}_{a}$ discussed in Remark 2 with $\widehat{G}=\widetilde{K}^{(d)} C_{y}$, we obtain the actual $\gamma$-value

$$
\gamma_{\widetilde{K}^{(d)}}=0.7525
$$

which exceeds the optimal value $\gamma_{\widetilde{G}_{s}}$ in only $0.79 \%$. Looking at the plots of singular values displayed in Fig. 12, it can be appreciated the good behavior of the decentralized velocity-feedback controller defined by the diagonal gain matrix $\widetilde{K}^{(d)}$ when compared with the optimal state-feedback controller.

Remark 9 Let us denote by $\mathcal{P}_{o}^{(d)}(\delta, \mu)$ the LMI optimization problem $\mathcal{P}_{o}$ in eq. (34) corresponding to the $L$ matrix $L_{\delta, \mu}^{(d)}$ and the zero-nonzero structure constraints given in eqs. (93) and (94). A suitable pair of parameter values $\delta^{*}$ and $\mu^{*}$ can be computed as follows: First, we select by inspection four boundary values

$$
\delta_{\ell}<\delta_{u}, \quad \mu_{\ell}<\mu_{u}
$$

for which the LMI optimization problems

$$
\mathcal{P}_{o}^{(d)}\left(\delta_{\ell}, \mu_{\ell}\right), \mathcal{P}_{o}^{(d)}\left(\delta_{\ell}, \mu_{u}\right), \mathcal{P}_{o}^{(d)}\left(\delta_{u}, \mu_{\ell}\right), \mathcal{P}_{o}^{(d)}\left(\delta_{u}, \mu_{u}\right)
$$


Table 1: Percentages of increment in the $\gamma$-values produced by the static velocity-feedback controllers with respect to the optimal value $\gamma_{\widetilde{G}_{s}}=0.7466$

\begin{tabular}{lcc}
\hline & \multicolumn{2}{c}{ Information structure } \\
\cline { 2 - 3 }$L$-matrix & Centralized & Decentralized \\
\hline$L=0$ & $0.94 \%$ & $4.05 \%$ \\
Advanced $L$ & $0.01 \%$ & $0.79 \%$ \\
\hline
\end{tabular}

are all feasible. Next, we consider the points

$$
\begin{gathered}
\delta_{i}=\delta_{\ell}+i\left(\delta_{u}-\delta_{\ell}\right) / n_{\delta}, i=0, \ldots, n_{\delta}, \\
\mu_{j}=\mu_{\ell}+j\left(\mu_{u}-\mu_{\ell}\right) / n_{\mu}, j=0, \ldots, n_{\mu},
\end{gathered}
$$

and solve the optimization problem

$$
\min _{i, j} \widetilde{\gamma}_{o}^{(d)}\left(\delta_{i}, \mu_{j}\right)
$$

where $\widetilde{\gamma}_{o}^{(d)}(\delta, \mu)$ is the optimal $\gamma$-value corresponding to the LMI optimization problem $\mathcal{P}_{o}^{(d)}(\delta, \mu)$. For our fivestory building control problem, we take the boundary values

$$
\delta_{\ell}=0.05, \delta_{u}=0.50, \mu_{\ell}=0.50, \mu_{u}=1.00
$$

and the grid of pairs $\left(\delta_{i}, \mu_{j}\right)$ defined by

$$
n_{\delta}=n_{\mu}=10 \text {. }
$$

In this case, we obtain the LMI optimization problems

$$
\mathcal{P}_{o}^{(d)}\left(\delta_{i}, \mu_{j}\right), i=0, \ldots, 10, j=0, \ldots, 10,
$$

which produce the minimum $\gamma$-value

$$
\widetilde{\gamma}_{o}^{(d)}\left(\delta^{*}, \mu^{*}\right)=0.7560
$$

for the parameter values $\delta^{*}$ and $\mu^{*}$ given in eq. (104).

Remark 10 Besides producing a suitable pair of parameter values $\delta^{*}$ and $\mu^{*}$ for the problem under consideration, the rudimentary computational strategy proposed in Remark 9 also illustrates the important role that can be played by the $L$-matrix in the design process. To compute the controller in eq. (108), we take advantage of the design flexibility provided by the $L$ matrix and explore a total number of 121 different controllers corresponding to the $L$-matrices

$$
L_{\delta_{i}, \mu_{j}}^{(d)}, \quad i=0, \ldots, 10, j=0, \ldots, 10,
$$

obtaining a fully decentralized velocity-feedback controller that exceeds the optimal $\gamma$-value attained by the optimal state-feedback controller in less than $1 \%$.
Moreover, it is worth highlighting that after selecting the boundary parameter values in eq. (114), the LMI optimization problems in eq. (116) are all feasible and the minimization problem in eq. (113) can be completed in about 8 seconds using an ordinary personal computer.

Remark 11 As indicated in Remark 5, the proposed design strategy for static output-feedback controllers relies on the following heuristic principle: the behavior of static output-feedback controllers with an almost optimal $\gamma$-value is similar to the behavior exhibited by the optimal state-feedback controller. Looking at the $\gamma$-value increments collected in Table 1 and the plots of singular values presented in Figs. 9-12, it becomes apparent that this heuristic principle holds for the different velocity-feedback controllers computed in Sects. 5 and 6. However, it should be noted that a smaller $\gamma$-value does not necessarily imply a uniformly better performance over the complete frequency range. Thus, for example, we can see in Table 1 that the minimum $\gamma$ value increment is attained by the centralized velocityfeedback controller with $L=\widetilde{L}$ computed in Sect. 5.3, whereas the maximum $\gamma$-value increment corresponds to the decentralized velocity-feedback controller with $L=0$ obtained in Sect.6.1. In contrast, looking at the graphics in Figs. 10 and 11, it can be appreciated that the decentralized controller has a better behavior in the frequency range $2-3 \mathrm{~Hz}$.

Remark 12 Analogously as it happened in the statefeedback case, solving the LMI optimization problems $\mathcal{P}_{o}$ and $\widehat{\mathcal{P}}_{o}$ using different computers or MATLAB versions can produce different control gain matrices. Consequently, the particular values obtained for the matrices $\widehat{K}, \widetilde{K}, \widehat{K}^{(d)}$ and $\widetilde{K}^{(d)}$ can differ significantly from those presented in eqs. (82), (89), (95) and (106), respectively. Additionally, if the $X$-matrix obtained in the design step (S1.1) is distinct from the matrix $\widetilde{X}_{s}$ presented in Fig. 5 , then the values of the matrix $\widetilde{L}$ in eq. (87), the values of the parameters $\delta^{*}$ and $\mu^{*}$ in eq. (104), and the matrix $L_{\delta^{*}, \mu^{*}}^{(d)}$ in eq. (105) will also be affected and, probably, a new set of boundary values $\delta_{\ell}, \delta_{u}, \mu_{\ell}, \mu_{u}$ will need to be selected in the optimization procedure described in Remark 9. However, it has to be highlighted that despite the differences observed in the control gain matrices, the obtained $\gamma$-values are very similar and the corresponding graphics of singular values are practically identical to those shown in Figs. 9-12.

\section{Numerical results}

To complement the frequency response information provided by Figs. 9-12, in this section we carry out 


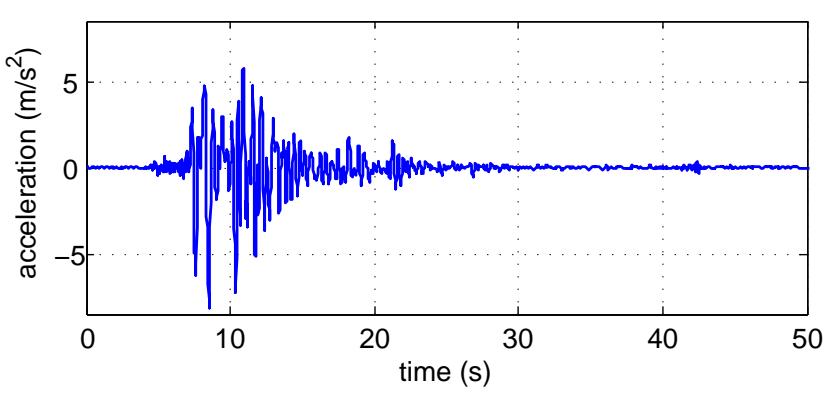

Figure 13: Full-scale North-South 1995 Kobe seismic record with an absolute acceleration peak of $8.18 \mathrm{~m} / \mathrm{s}^{2}$

numerical simulations of the five-story building vibrational response using two different ground acceleration inputs: (i) the full scale North-South El Centro 1940 seismic record (see Fig. 7), employed previously in Sect. 5.1 in the performance assessment of the optimal state-feedback controller and (ii) the full scale NorthSouth Kobe 1995 seismic record displayed in Fig. 13, which is a larger seismic disturbance with an absolute acceleration peak of $8.18 \mathrm{~m} / \mathrm{s}^{2}$.

In Fig. 14, we present the maximum absolute interstory drifts and maximum absolute control efforts corresponding to the centralized velocity-feedback controllers designed in Sects. 5.2 and 5.3. The graphics corresponding to the decentralized velocity-feedback controllers computed in Sects. 6.1 and 6.2 are displayed in Fig. 15. In the different subfigures, the green lines with triangles represent the graphics associated with velocity-feedback controllers designed with a null $L$ matrix, which are denoted as "velocity-feed. $L=0$ " in the legends, and the red lines with asterisks show the graphics pertaining to velocity-feedback controllers computed using more advanced $L$-matrix choices, which are denoted as "velocity-feed. adv. $L$ " in the legends. Additionally, to facilitate proper references for the performance assessment of the proposed velocityfeedback controllers, plots corresponding to the uncontrolled building and the optimal state-feedback $H_{\infty}$ controller have also been included, using black lines with rectangles and blue lines with circles, respectively.

For the North-South El Centro 1940 seismic disturbance, the graphics in Figs. 14(a) and 14(b) show that the behavior of the centralized velocity-feedback controller defined by the output gain matrix $\widetilde{K}$ in eq. (89) is very similar to the behavior exhibited by the optimal state-feedback controller. Looking at the graphics in Figs. 14(c) and 14(d), we can also see that the behavior of both controllers is practically the same in the case of the North-South Kobe 1995 seismic disturbance. For the centralized velocity-feedback controller defined by the output gain matrix $\widehat{K}$ given in eq. (82), the graphics in Fig. 14 indicate that this controller, designed with the simplified choice $L=0$, presents an overall good behavior but the differences with respect to the optimal state-feedback controller are quite significant, specially for the larger seismic disturbance.

Regarding the decentralized controllers, the graphics in Fig. 15 show that the performance of the decentralized velocity-feedback controller defined by the diagonal output gain matrix $\widetilde{K}^{(d)}$ in eq. (106) is certainly remarkable. Looking at the plots in Figs. 15(a) and 15(b), we can see that, for the North-South El Centro 1940 seismic disturbance, the decentralized controller computed with the advanced $L$-matrix $L_{\delta^{*}, \mu^{*}}^{(d)}$ attains practically the same level of reduction in the interstory drift peak-values as the optimal state-feedback controller, presenting only a small increment of the control effort peak-value in the first actuation device. For the North-South Kobe 1995 seismic record, the differences increase but the behavior of the decentralized velocityfeedback controller is still quite close to the behavior exhibited by the optimal state-feedback controller. The plots in Fig. 15 also indicate that the decentralized velocity-feedback controller defined by the diagonal output gain matrix $\widehat{K}^{(d)}$ in eq. (95) and computed with a null $L$-matrix has a remarkably good behavior. This decentralized controller attains levels of reduction in the interstory drift peak-values that are quite similar to those achieved by the optimal state-feedback controller, requiring control efforts with smaller peak values for the upper four actuation devices but a significantly larger peak value in the first actuation device.

Remark 13 It has to be highlighted that the decentralized velocity-feedback controllers defined by the diagonal output gain matrices $\widetilde{K}^{(d)}$ and $\widehat{K}^{(d)}$ can be implemented by means of a system of passive linear dampers with no sensors, no communication system and null power consumption [Palacios-Quiñonero et al. (2012c)]. The performance of the proposed decentralized velocity-feedback controllers, illustrated by the plots in Fig. 15, should be evaluated in the light of this important property.

\section{Conclusions and future directions}

In this work, a two-step procedure to design static output-feedback controllers has been proposed. This novel design methodology, based on recent theoretical results presented in Rubió-Massegú et al. (2013b), can be applied to a wide variety of control problems for which satisfactory state-feedback controllers can be obtained using a linear matrix inequality formulation. In particular, the new approach has proved to be effec- 
tive in providing suitable solutions to large-scale control problems with information constraints. The design procedure includes the choice of a matrix $L$ that plays an important role in the effectiveness of the method. In some cases, positive results can be achieved with the simplified choice $L=0$. Also, more sophisticated $L$-matrices can be obtained by applying the theoretical results presented in Palacios-Quiñonero et al. (2014b). In this paper, an advanced $L$-matrix choice specially devised for fully decentralized velocity-feedback $H_{\infty}$ controller design is provided.

To illustrate the flexibility and effectiveness of the two-step design procedure, a set of five different $H_{\infty}$ controllers for the seismic protection of a five-story building has been designed. This set includes a centralized state-feedback controller, which uses the full state as feedback information and is taken as a reference in the performance assessment; two centralized static velocity-feedback controllers, which can operate using only the interstory velocities as feedback information; and two fully decentralized static velocity-feedback controllers, which can be implemented by means of a system of passive linear dampers. To assess the performance of the static velocity-feedback controllers, the building frequency response has been investigated for the different control configurations. Also, numerical simulations of the building vibrational response have been conducted, using the full scale North-South El Centro 1940 and North-South Kobe 1995 seismic records as ground acceleration inputs. The numerical data indicate that, despite the restricted access to the state information, the behavior of the proposed static velocity-feedback controllers is similar to the behavior exhibited by the optimal state-feedback controller. Moreover, improved performance levels can be attained with advanced choices of the $L$-matrix.

The positive results obtained to date, clearly indicate that further research effort should be addressed to apply the proposed design methodology in more complex control problems, such as simultaneous stabilization [Shi and Qi (2009)], switching systems [Hou et al. (2012); Attia et al. (2012); Xiang et al. (2014); Li et al. (2014)], networked control [Yang et al. (2011); Zhang and Wang (2012)], robust control [Toscano and Lyonnet (2010); Vaselý et al. (2011); Dong and Yang (2013); Aouaouda et al. (2014)], fuzzy systems [Ho et al. (2012); Zhang et al. (2014)] and finite frequency control [Chen et al. (2010); Wang et al. (2014)]].

\section{Acknowledgments}

This work was partially supported by the Spanish Ministry of Economy and Competitiveness through the grant DPI2012-32375/FEDER; by a Grant from Ice- land, Liechtenstein and Norway through the EEA Financial Mechanism, operated by Universidad Complutense de Madrid; and by the Norwegian Center of Offshore Wind Energy (NORCOWE) under Grant 193821/S60 from the Research Council of Norway $(\mathrm{RCN})$. NORCOWE is a consortium with partners from industry and science, hosted by Christian Michelsen Research.

\section{References}

Amato, F., Cosentino, C., and Merola, A. Sufficient conditions for finite-time stability and stabilization of nonlinear quadratic systems. IEEE Transactions on Automatic Control, 2010. 55(2):430-434. doi:10.1109/TAC.2009.2036312.

Aouaouda, S., Chadli, M., and Karimi, H. Robust static output-feedback controller design against sensor failure for vehicle dynamics. IET Control Theory and Applications, 2014. 8(9):728-737. doi:10.1049/iet-cta.2013.0709.

Attia, S., Salhi, S., and Ksouri, M. Static switched output feedback stabilisation for linear discrete-time switched systems. International Journal of Innovative Computing, Information and Control, 2012. 8(5):3203-3213.

Bakka, T. and Karimi, H. $H_{\infty}$ static outputfeedback control design with constrained information for offshore wind turbine system. Journal of the Franklin Institute, 2013. 350(8):2244-2260. doi:10.1016/j.jfranklin.2013.05.028.

Bakka, T., Karimi, H., and Christiansen, S. Linear parameter-varying modelling and control of an offshore wind turbine with constrained information. IET Control Theory and Applications, 2014. 8(1):2229. doi:10.1049/iet-cta.2013.0480.

Balas, G., Chiang, R., Packard, A., and Safonov, M. MATLAB Robust Control Toolbox 3. User's Guide. The MathWorks, Inc., Natick, USA, 2011.

Ballesteros, P., Shu, X., Heins, W., and Bohn, C. Reduced-order two-parameter pLPV controller for the rejection of nonstationary harmonically related multisine disturbances. In Proceedings of the 2013 European Control Conference (ECC). Zürich, Switzerland, pages 1835-1842, 2013.

Bara, G. and Boutayeb, M. Static output feedback stabilization with $H_{\infty}$ performance for linear discrete-time systems. IEEE Transactions on Automatic Control, $2005 . \quad 50(2): 250-254$. doi:10.1109/TAC.2004.841922. 
Boyd, S., Ghaoui, L. E., Feron, E., and Balakrishnan, V. Linear Matrix Inequalities in System and Control Theory. SIAM Studies in Applied Mathematics, Philadelphia, USA, 1994.

Cao, Y.-Y., Lam, J., and Sun, Y.-X. Static output feedback stabilization: an ILMI approach. Automatica, 1998. 34(12):1641-1645. doi:10.1016/S00051098(98)80021-6.

Chen, A. and Wang, J. Delay-dependent $L_{2^{-}}$ $L_{\infty}$ control of linear systems with multiple timevarying state and input delays. Nonlinear Analysis: Real World Applications, 2012. 13(1):486-496. doi:10.1016/j.nonrwa.2011.08.006.

Chen, X.-B. and Stanković, S. Decomposition and decentralized control of systems with multi-overlapping structure. Automatica, 2005. 41(10):1765-1772. doi:10.1016/j.automatica.2005.01.020.

Chen, Y., Zhang, W., and Gao, H. Finite frequency $H_{\infty}$ control for building under earthquake excitation. Mechatronics, 2010. 20(1):128-142. doi:10.1016/j.mechatronics.2009.11.001.

Chopra, A. Dynamics of Structures. Theory and Applications to Earthquake Engineering. Prentice Hall, Upper Saddle River, New Jersey, USA, 3rd edition, 2007.

Crusius, C. and Trofino, A. Sufficient LMI conditions for output feedback control problems. IEEE Transactions on Automatic Control, 1999. 44(5):10531057. doi:10.1109/9.763227.

Dhawan, A. and Kar, H. An improved LMIbased criterion for the design of optimal guaranteed cost controller for 2-D discrete uncertain systems. Signal Processing, 2011. 91(4):1032-1035. doi:10.1016/j.sigpro.2010.07.014.

Dong, J. and Yang, G.-H. Robust static output feedback control synthesis for linear continuous systems with polytopic uncertainties. Automatica, 2013. 49(6):1821-1829. doi:10.1016/j.automatica.2013.02.047.

Du, H., Zhang, N., and Naghdy, F. Actuator saturation control of uncertain structures with input time delay. Journal of Sound and Vibration, 2011. 330(1819):4399-4412. doi:10.1016/j.jsv.2011.04.025.

Feng, J.-E., Lam, J., Li, P., and Shu, Z. Decay rate constrained stabilization of positive systems using static output feedback. International Journal of Robust and Nonlinear Control, 2011. 21(1):44-54. doi:10.1002/rnc.1575.
Gadewadikar, J., Lewis, F., Xie, L., Kucera, V., and Abu-Khalaf, M. Parameterization of all stabilizing $H_{\infty}$ static state-feedback gains: application to output-feedback design. Automatica, 2007. 43(9):1597-1604. doi:10.1016/j.automatica.2007.02.005.

Ho, W.-H., Chen, S.-H., Chen, I.-T., Chou, J.-H., and Shu, C.-C. Design of stable and quadraticoptimal static output feedback controllers for TSfuzzy-model-based control systems: an integrative computational approach. International Journal of Innovative Computing, Information and Control, 2012. 8(1):403-418.

Hou, L., Zong, G., and Wu, Y. Finite-time control for switched delay systems via dynamic output feedback. International Journal of Innovative Computing, Information and Control, 2012. 8(7):4901-4913.

Huang, L. and Mao, X. Robust delayed-statefeedback stabilization of uncertain stochastic systems. Automatica, 2009. 45(5):1332-1339. doi:10.1016/j.automatica.2009.01.004.

Karimi, H., Palacios-Quiñonero, F., Rossell, J., and Rubió-Massegú, J. Sequential design of multioverlapping controllers for structural vibration control of tall buildings under seismic excitation. Proceedings of the Institution of Mechanical Engineers, Part I: Journal of Systems and Control Engineering, 2013. 227(2):176-183. doi:10.1177/0959651812464026.

Kurata, N., Kobori, T., Takahashi, M., Niwa, N., and Midorikawa, H. Actual seismic response controlled building with semi-active damper system. Earthquake Engineering and Structural Dynamics, 1999. 28(11):1427-1447.

Li, Z., Gao, H., and Karimi, H. Stability analysis and $H_{\infty}$ controller synthesis of discretetime switched systems with time delay. Systems and Control Letters, 2014. 66:85 - 93. doi:10.1016/j.sysconle.2013.12.010.

Liu, A., Yu, L., and Zhang, W. $H_{\infty}$ control for network-based systems with time-varying delay and packet disordering. Journal of the Franklin Institute, 2011. 348(5):917-932. doi:10.1016/j.jfranklin.2011.03.002.

Lunze, J. Feedback Control of Large-Scale Systems. Prentice Hall, Upper Saddle River, NJ, USA, 1992.

Moerder, D. and Calise, A. Convergence of a numerical algorithm for calculating optimal output feedback gains. IEEE Transactions on Automatic Control, $1985 . \quad 30(9): 900-903$. doi:10.1109/TAC.1985.1104073. 
Oishi, Y. and Fujioka, H. Stability and stabilization of aperiodic sampled-data control systems using robust linear matrix inequalities. Automatica, 2010. 46(8):1327-1333. doi:10.1016/j.automatica.2010.05.006.

Oufroukh, N. and Mammar, S. Integrated driver copilote approach for vehicle lateral control. In Proceedings of the 2014 IEEE Intelligent Vehicles Symposium. Dearborn, USA, pages 1163-1168, 2014. doi:10.1109/IVS.2014.6856519.

Palacios-Quiñonero, F., Karimi, H., Rubió-Massegú, J., and Rossell, J. Passive-damping design for vibration control of large structures. In Proceedings of the 10th International Conference on Control and Automation (IEEE ICCA 2013). Hangzhou, China, pages 33-38, 2013. doi:10.1109/ICCA.2013.6565018.

Palacios-Quiñonero, F., Rodellar, J., and Rossell, J. Sequential design of multi-overlapping controllers for longitudinal multi-overlapping systems. Applied Mathematics and Computation, 2010. 217(3):11701183. doi:10.1016/j.amc.2010.01.130.

Palacios-Quiñonero, F., Rossell, J., and Karimi, H. Semi-decentralized strategies in structural vibration control. Modeling, Identification and Control, 2011. 32(2):57-77. doi:10.4173/mic.2011.2.2.

Palacios-Quiñonero, F., Rubió-Massegú, J., Rossell, J., and Karimi, H. Discrete-time multioverlapping controller design for structural vibration control of tall buildings under seismic excitation. Mathematical Problems in Engineering, 2012a. 2012:1-20. doi:10.1155/2012/636878.

Palacios-Quiñonero, F., Rubió-Massegú, J., Rossell, J., and Karimi, H. Discrete-time static outputfeedback semi-decentralized $H_{\infty}$ controller design: an application to structural vibration control. In Proceedings of the 2012 American Control Conference. Montreal, Canada, pages 6126-6131, 2012b. doi:10.1109/ACC.2012.6314816.

Palacios-Quiñonero, F., Rubió-Massegú, J., Rossell, J., and Karimi, H. Optimal passive-damping design using a decentralized velocity-feedback $H_{\infty}$ approach. Modeling, Identification and Control, 2012c. 33(3):87-97. doi:10.4173/mic.2012.3.1.

Palacios-Quiñonero, F., Rubió-Massegú, J., Rossell, J., and Karimi, H. Discrete-time static output-feedback $H_{\infty}$ controller design for vehicle suspensions. In Proceedings of the 2014 International Conference on Mechatronics and Control (ICMC). Jinzhou, China, pages 2346-2351, 2014a.
Palacios-Quiñonero, F., Rubió-Massegú, J., Rossell, J., and Karimi, H. Feasibility issues in static output-feedback controller design with application to structural vibration control. Journal of the Franklin Institute, 2014b. 351(1):139-155. doi:10.1016/j.jfranklin.2013.08.011.

Palacios-Quiñonero, F., Rubió-Massegú, J., Rossell, J., and Karimi, H. Optimal design of complex passive-damping systems for vibration control of large structures: an energy-to-peak approach. Abstract and Applied Analysis, 2014c. 2014:1-9. doi:10.1155/2014/510236.

Palacios-Quiñonero, F., Rubió-Massegú, J., Rossell, J., and Karimi, H. Static output-feedback controller design for structural vibration control: a twostep LMI approach. In Proceedings of the Sixth World Conference on Structural Control and Monitoring (6WCSCM). Barcelona, Spain, pages 19021913, 2014d.

Palacios-Quiñonero, F., Rubió-Massegú, J., Rossell, J., and Karimi, H. Vibration control for adjacent structures using local state information. Mechatronics, 2014e. 24(4):336-344. doi:10.1016/j.mechatronics.2013.08.001.

Prempain, E. and Postlethwaite, I. Static output feedback stabilisation with $H_{\infty}$ performance for a class of plants. Systems and Control Letters, 2001. 43(3):159-166. doi:10.1016/S0167-6911(01)00087-1.

Rossell, J., Palacios-Quiñonero, F., and Rodellar, J. Semi-decentralized output feedback $H_{\infty}$ control strategy for large building structures. In Proceedings of the 5th World Conference on Structural Control and Monitoring (5WCSCM). Shinjuku, Japan, 2010.

Rubió-Massegú, J., Palacios-Quiñonero, F., and Rossell, J. Decentralized static output-feedback $H_{\infty}$ controller design for buildings under seismic excitation. Earthquake Engineering and Structural Dynamics, 2012. 41(7):1199-1205. doi:10.1002/eqe.1167.

Rubió-Massegú, J., Palacios-Quiñonero, F., Rossell, J., and Karimi, H. Static output-feedback control for vehicle suspensions: a single-step linear matrix inequality approach. Mathematical Problems in Engineering, 2013a. 2013:1-12. doi:10.1155/2013/907056.

Rubió-Massegú, J., Palacios-Quiñonero, F., Rossell, J., and Karimi, H. Static output-feedback controller design for vehicle suspensions: an effective two-step computational approach. IET Control 
Theory and Applications, 2014. 8(15):1566-1574. doi:10.1049/iet-cta.2013.1129.

Rubió-Massegú, J., Rossell, J., Karimi, H., and Palacios-Quiñonero, F. Static outputfeedback control under information structure constraints. Automatica, 2013b. 49(1):313-316. doi:10.1016/j.automatica.2012.10.012.

Shi, H.-B. and Qi, L. Static output feedback simultaneous stabilisation via coordinates transformations with free variables. IET Control Theory and Applications, 2009. 3(8):1051-1058. doi:10.1049/ietcta.2008.0185.

Šiljak, D. Decentralized Control of Complex Systems. Academic Press, New York, USA, 1991.

Syrmos, V., Abdallah, C., Dorato, P., and Grigoriadis, K. Static output feedback - A survey. Automatica, 1997. 33(2):125-137. doi:10.1016/S00051098(96)00141-0.

Toscano, R. A simple method to find a robust output feedback controller by random search approach. ISA Transactions, 2006. 45(1):35-44. doi:10.1016/S00190578(07)60063-9.

Toscano, R. and Lyonnet, P. Robust static output feedback controller synthesis using Kharitonov's theorem and evolutionary algorithms. Information Sciences, $2010 . \quad$ 180(10):2023-2028. doi:10.1016/j.ins.2010.01.009.

Vaselý, V., Rosinová, D., and Kucera, V. Robust static output feedback controller LMI based design via elimination. Journal of the Franklin Institute, 2011. 348(9):2468-2479. doi:10.1016/j.jfranklin.2011.04.020.

Wang, R., Jing, H., Yan, F., Karimi, H., and Chen, N. Optimization and finite-frequency $H_{\infty}$ control of active suspensions in in-wheel motor driven electric ground vehicles. Journal of the Franklin Institute, 2014. In press. doi:10.1016/j.jfranklin.2014.05.005.
Wang, Y., Lynch, J., and Law, K. Decentralized $H_{\infty}$ controller design for large-scale civil structures. Earthquake Engineering and Structural Dynamics, 2009. 38(3):377-401. doi:10.1002/eqe.862.

Wang, Z., Liu, Y., Wei, G., and Liu, X. A note on control of a class of discrete-time stochastic systems with distributed delays and nonlinear disturbances. Automatica, 2010. 46(3):543-548. doi:10.1016/j.automatica.2009.11.020.

Xiang, M., Xiang, Z., and Karimi, H. Stabilization of positive switched systems with time-varying delays under asynchronous switching. International Journal of Control, Automation and Systems, 2014. 12(5):939-947. doi:10.1007/s12555-013-0486-x.

Yang, R., Shi, P., Liu, G.-P., and Gao, H. Networkbased feedback control for systems with mixed delays based on quantization and dropout compensation. Automatica, 2011. 47(12):2805-2809. doi:10.1016/j.automatica.2011.09.007.

Zečević, A. and Šiljak, D. Design of robust static output feedback for large-scale systems. IEEE Transactions on Automatic Control, 2004. 49(11):2040-2044. doi:10.1109/TAC.2004.837542.

Zečević, A. and Šiljak, D. Control of Complex Systems. Structural Constraints and Uncertainty. Springer, 2010. doi:10.1007/978-1-4419-1216-9.

Zhang, D. and Wang, X. Static output feedback control of networked control systems with packet dropout. International Journal of Systems Science, 2012. 43(4):665-672. doi:10.1080/00207721.2010.517873.

Zhang, Y., Shi, P., Nguang, S. K., Karimi, H., and Agarwal, R. Robust finite-time fuzzy $H_{\infty}$ control for uncertain time-delay systems with stochastic jumps. Journal of the Franklin Institute, 2014. 351(8):42114229. doi:10.1016/j.jfranklin.2014.04.004. 

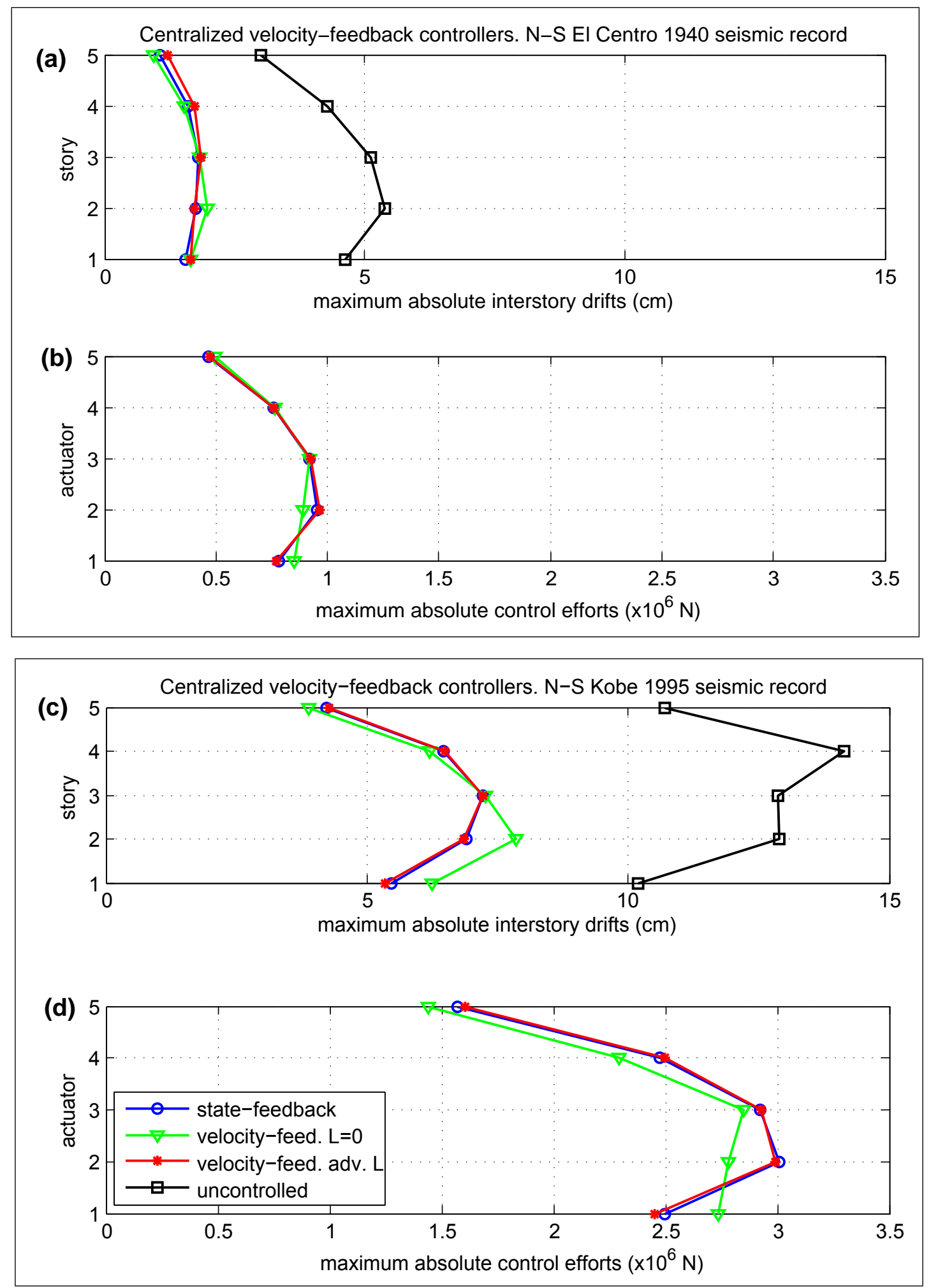

Figure 14: Maximum absolute interstory drifts and maximum absolute control efforts corresponding to the centralized velocity-feedback controllers defined by the control gain matrices $\widehat{K}$ (green line with triangles) and $\widetilde{K}$ (red line with asterisks). The plots corresponding to the uncontrolled building (black line with rectangles) and the optimal state-feedback $H_{\infty}$ controller (blue line with circles) are also included as a reference. The full scale North-South El Centro 1940 seismic record has been used as ground acceleration input to obtain the plots in Figs. (a) and (b). For the plots in Figs. (c) and (d), the full scale North-South Kobe 1995 seismic record has been taken as ground acceleration input 

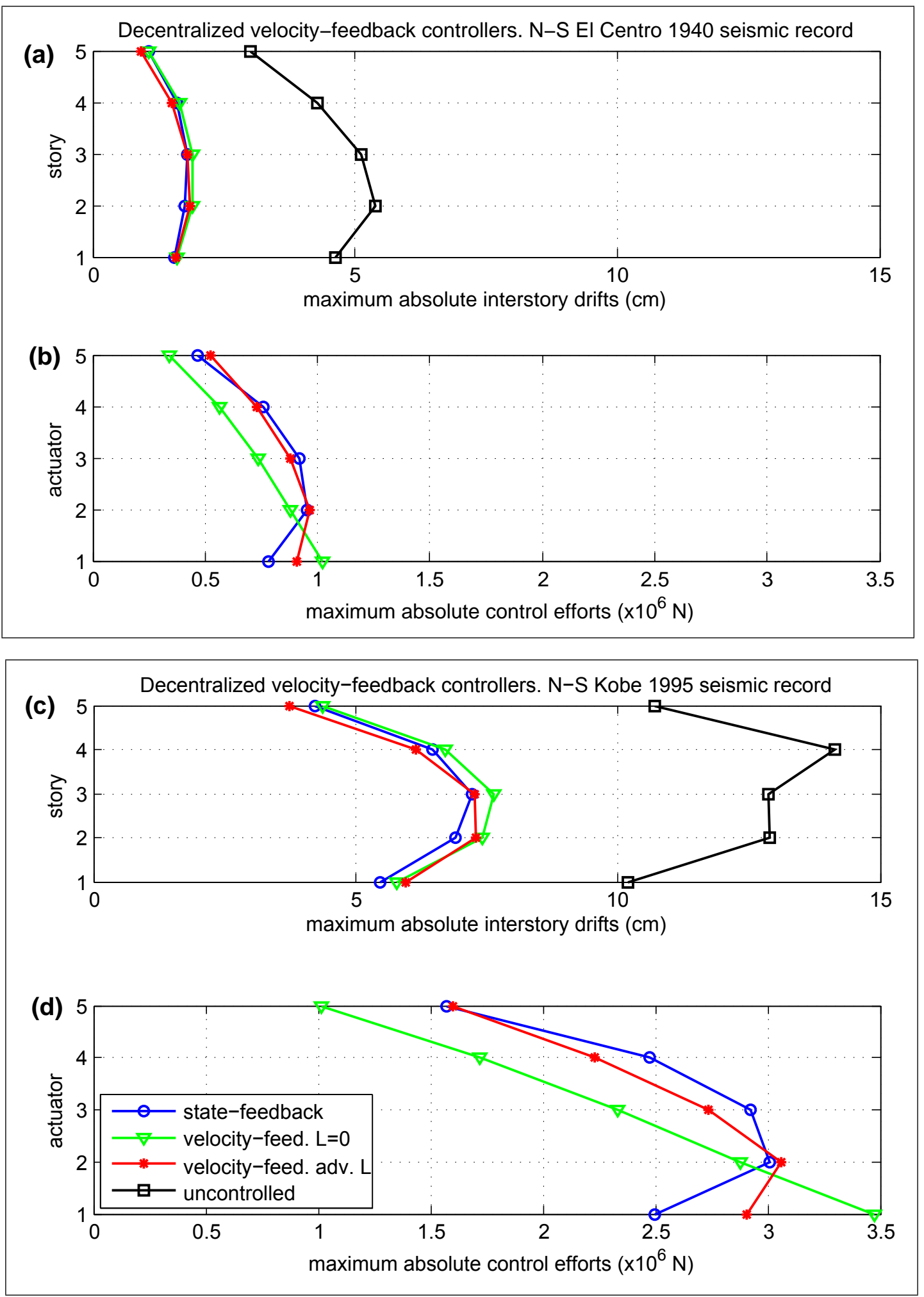

Figure 15: Maximum absolute interstory drifts and maximum absolute control efforts corresponding to the decentralized velocity-feedback controllers defined by the diagonal control gain matrices $\widehat{K}^{(d)}$ (green line with triangles) and $\widetilde{K}^{(d)}$ (red line with asterisks). The plots corresponding to the uncontrolled building (black line with rectangles) and the optimal state-feedback $H_{\infty}$ controller (blue line with circles) are also included as a reference. The full scale North-South El Centro 1940 seismic record has been used as ground acceleration input to obtain the plots in Figs. (a) and (b). For the plots in Figs. (c) and (d), the full scale North-South Kobe 1995 seismic record has been taken as ground acceleration input 